Report: UCRL-TR-201506

\title{
Static and Dynamic Criticality: Are They Different?
}

\author{
by \\ Dermott E. Cullen \\ Christopher J. Clouse \\ Richard Procassini \\ and \\ Robert C. Little
}

November 22, 2003

U.S. Department of Energy

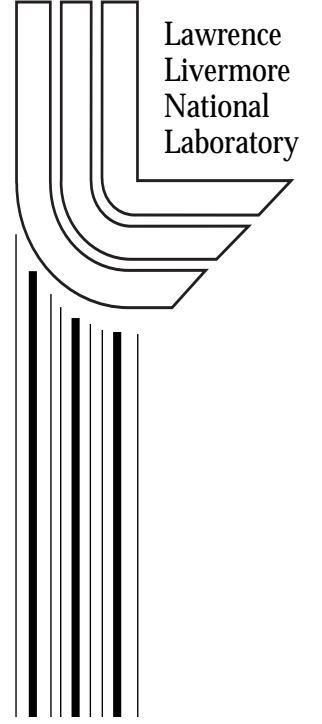

Approved for public release; further dissemination unlimited. 
This work was produced at the University of California, Lawrence Livermore National Laboratory (UC LLNL) under contract no. W-7405-ENG-48 (Contract 48) between the U.S. Department of Energy (DOE) and The Regents of the University of California (University) for the operation of UC LLNL. The rights of the Federal Government are reserved under Contract 48 subject to the restrictions agreed upon by the DOE and University as allowed under DOE Acquisition Letter 97-1.

\title{
DISCLAIMER
}

This work was prepared as an account of work sponsored by an agency of the United States Government. Neither the United States Government nor the University of California nor any of their employees, makes any warranty, express or implied, or assumes any liability or responsibility for the accuracy, completeness, or usefulness of any information, apparatus, product, or process disclosed, or represents that its use would not infringe privately-owned rights. Reference herein to any specific commercial products, process, or service by trade name, trademark, manufacturer or otherwise does not necessarily constitute or imply its endorsement, recommendation, or favoring by the United States Government or the University of California. The views and opinions of authors expressed herein do not necessarily state or reflect those of the United States Government or the University of California, and shall not be used for advertising or product endorsement purposes.

\section{NOTIFICATION OF COMMERCIAL USE}

Commercialization of this product is prohibited without notifying the Department of Energy (DOE) or Lawrence Livermore National Laboratory

\section{REPRODUCTION}

This report has been reproduced directly from the best available copy.

\author{
Available to DOE and DOE contractors from the \\ Office of Scientific and Technical Information \\ P.O. Box 62, Oak Ridge, TN 37831 \\ Prices available from (423) 576-8401 \\ http://apollo.osti.gov/bridge/ \\ Available to the public from the \\ National Technical Information Service \\ U.S. Department of Commerce \\ 5285 Port Royal Rd., \\ Springfield, VA 22161 \\ http://www.ntis.gov/
}

\section{OR}

Lawrence Livermore National Laboratory

Technical Information Department's Digital Library

http://www.Ilnl.gov/tid/Library.html 
$\underline{\text { UCRL-TR-201506 }}$

$\underline{\text { Static and Dynamic Criticality: Are they different? }}$

by

Dermott E. Cullen

Christopher J. Clouse

Richard Procassini

Lawrence Livermore National Laboratory

and

Robert C. Little

Los Alamos National Laboratory

contact

Dermott E. Cullen

University of California

Lawrence Livermore National Laboratory

L-159

P.O. Box 808

Livermore, CA 94550

E.Mail: cullen1@Ilnl.gov

Website: $\underline{\text { http://www.llnl.gov/cullen1 }}$

November 22, 2003

\section{Overview}

Let us start by stating that this paper does not contain anything new. It only contains material that has been known for decades, but which is periodically forgotten. As such this paper is intended merely to refresh people's memories. We will also mention that this paper is an example of the occasional discrepancy between textbook methodologies and real world applications, in the sense that the conclusions reached here contradict what it says in most textbooks, i.e., most textbooks incorrectly interpret the methods presented here, particularly with respect to the use of importance sampling to maintain population control.

This paper is not intended as a general tutorial on criticality calculations. It is intended only to clarify the accuracy of various methods for solving criticality problems, such as a true time dependent dynamic calculation, versus an alpha or $\mathrm{K}$ static calculation. In particular, we address the long standing controversy between users of the TART code [1] with the dynamic method, and users of the MCNP code [2] with the alpha static method. In this paper we will prove which methods are accurate and inaccurate.

Let us first give you the answer: what the TART and MCNP codes do is equivalent. A dynamic calculation is an unbiased calculation of the actual time dependence of a system, 
i.e., a dynamic calculation produces accurate answers. For criticality calculations the alpha static method is exactly equivalent to a dynamic calculation, i.e., an alpha static calculation produces accurate answers. In contrast, the $\mathrm{K}$ static method, which is taught in most schools and described in many textbooks, is accurate for systems that are close to critical, but for systems far from critical it introduces approximations that bias the neutron flux in energy and space, and as such do not produce the same results as the unbiased dynamic method, i.e., for systems far from critical the K static method produces incorrect answers, or more precisely correctly solves a different problem then the one we are interested in, as explained below. We will show that the results of dynamic and $\mathrm{K}$ static calculations only agree for critical systems $(\mathrm{K}$-eff $=1)$. For other systems, the results differ with the differences between the results increasing the further a system is from criticality.

Since this is the entire point of this paper let us repeat the conclusions,

1) dynamic and alpha static criticality calculations are identical, so that if properly used TART with the dynamic method and MCNP with the alpha static method, should produce the same answers.

2) The textbook K static method that we were all taught in school, is generally only accurate for systems that are close to critical; as we will see below, in other cases this method can be very inaccurate.

\section{Where can the K Static Method be Used?}

The K static method described in this paper has been successfully used for criticality calculations for over 50 years. It has been the workhorse of the criticality business, and has been widely documented in virtually every textbook on the subject, and we are sure that it will continue to be just a widely used in the future. Here we point out that when properly used this method is accurate, practical and very economical for use in criticality calculation.

But we also document where this method can be "properly" used, i.e., if you misuse any method the answers you get are questionable. In the case of the $\mathrm{K}$ static method it is very accurate for systems close to critical, i.e., K-eff $\sim 1$. It is also very effective if you wish merely to qualitatively define whether a system is sub or super-critical, as well as for monitoring the approach of systems to or from an exactly critical state. However, if you are interested in systems that are far from critical, the $\mathrm{K}$ static method may not be accurate, and the difference between the true and accuracy solution and the $\mathrm{K}$ static method solution grows as a system becomes progressively further from critical.

\section{Statement of the Problem}

Here we will be interested in time dependent neutron transport through time independent media, i.e., the material that the neutrons transport though is not changing with time. Also, initially we will not consider delayed neutrons. Later we briefly discuss time dependent media and delayed neutrons. With these restrictions the Boltzmann equation of interest is, 


$$
\begin{array}{ll}
\frac{1}{v} \frac{\partial N}{\partial t}+\Omega^{*} \nabla N+\Sigma t^{*} N=\iint[<v>* \Sigma f+\Sigma s] F\left(E^{\prime}, \Omega^{\prime}->E, \Omega\right) N d E^{\prime} d \Omega^{\prime}+S \\
N(r, \Omega, E, t) & \text { - neutron flux } \\
S(r, \Omega, E, t) & \text { - flux independent source } \\
\Sigma t(r, E) & \text { - total cross section } \\
\Sigma f(r, E) & \text { - fission cross section } \\
\Sigma s(r, E) & \text { - scatter cross section } \\
<v(r, E)> & \text { - average number of neutrons emitted per fission } \\
F\left(r, E^{\prime} ; E^{\prime}, \Omega^{\prime}->E, \Omega\right) & \text { - probability of transfer from }\left(E^{\prime}, \Omega^{\prime}\right) \text { to }(E, \Omega) \\
v & \text { - neutron speed (not velocity; speed) }
\end{array}
$$

Here the production term $\langle v\rangle \Sigma f$ actually represents all neutron production, i.e., it also includes $(n, 2 n),(n, 3 n)$, etc.

Let us repeat that the neutron flux is time dependent, but the material through which the neutrons are passing is not time dependent. Therefore, in our equation all of the material properties, such as cross sections, are independent of time. Also in this equation because delayed neutrons are not included, everything happens instantaneously, e.g., all neutrons emitted due to fission are emitted at the instant that the fission occurs; see the appendix for the appropriate equations to use when delayed neutrons are included.

\section{General Solution}

Today our computers are large enough and fast enough to allow us to solve time dependent neutron transport problems using true analog methods, without placing any, or at least few, restrictions on the calculation. In this type of calculation we can start from any initial (in time) source distribution, $S(r, \Omega, E, 0)$, and follow the evolution of the flux $N(r, \Omega, E, t)$ in all dimensions as it either increases, if the system is super-critical, or decreases, if the system is sub-critical. We cannot follow this evolution forever in time, but at least today's computers with gigabytes of memory allow us to follow this growth or decline by factors of a million, or even at billion, change in neutron population. This is usually sufficient to allow us to observe the actual time evolution of the system from its initial, time zero, distribution, to its eventual evolution into its fundamental mode distribution. Following this procedure, we can obtain a very general solution at least for some finite period of time.

The criticality methods that we describe below historically have not used this approach. In criticality methods we are interested not in a general solution, but rather in the solution only after the system has relaxed into its fundamental mode, and unlike the general solution, described above, these methods use importance sampling to control (limit) the neutron population. An important objective of this paper is to determine whether or not criticality methods produce the same answers as a true analog calculation. 


\section{Fundamental Mode Solution}

In the above equation we have not put any restriction on the flux independent source distribution; in principle we can solve this equation for any source distribution. But in this paper we are interested in solving this equation as a criticality problem. The way that computer codes solve this problem is to start with an arbitrary source, $S(r, \Omega, E, 0)$, at time zero and follow the evolution of the neutron flux in time. We assume that eventually this will result in the system relaxing into its fundamental mode. The subsequent behavior of the system is then characterized by a simple, single exponential variation of the flux with time, $N_{0}(t)=N_{0}(0) \operatorname{Exp}\left(\alpha^{*} t\right)$ : for a super-critical system the flux will exponentially increase $(\alpha>0)$, for a sub-critical system it will decrease $(\alpha<0)$, and for a critical system it will remain constant $(\alpha=0)$.

We will not go into details of the fundamental mode, but briefly we assume that starting from any neutron source distribution $S(r, E, \Omega, 0)$ we can define the time dependent flux distribution as the sum over modes, or terms, each of which has its own characteristic time dependence,

$N(r, E, \Omega, t)=\sum_{j=0}^{\infty} N_{j}(r, E, \Omega) * \operatorname{Exp}\left[\alpha_{j} * t\right]$

For a sub-critical system all of the values of $\alpha_{j}$ are negative and in this sum are in increasing absolute value order versus $\mathrm{j}$. This means that with time all of the other modes will die away faster and eventually leave only one mode, the fundamental mode, to define the subsequent time dependence of the system,

$N(r, E, \Omega, t)=N_{0}(r, E, \Omega) * \operatorname{Exp}\left[\alpha_{0} * t\right] ; \alpha_{0}<0$

For a super-critical system we assume one of the values of $\alpha$ is positive and all of the others are negative. Which means that with time all of the other modes will die away and eventually leave only one mode, the fundamental mode, to define the subsequent time dependence of the system,

$N(r, E, \Omega, t)=N_{0}(r, E, \Omega) * \operatorname{Exp}\left[\alpha_{0} * t\right] ; \alpha_{0}>0$

We can see that this assumes that after some period of time the distribution in (space, energy, direction) becomes stationary and, subsequently, the distribution only varies in magnitude as $\operatorname{Exp}[\alpha 0 * t]$.

Without explicitly writing the source distribution as a sum of modes, in a criticality calculation we assume that this is what will happen to our distribution. So that starting from any initial source distribution, the criticality methods described below assume that when we iterate over time, the system will eventually relax into its fundamental mode, 
and subsequent time dependence will show a simple, single exponential variation with time.

Below we will see that the static criticality methods, where time is not explicitly included, assume that the same relaxation into its fundamental mode will happen after a number of generations, and we also assume that, subsequently, each generation will have the same (space, energy, direction) distribution and differ only in magnitude depending on K-eff.

\section{Non-Fundamental Mode Solution}

It should be mentioned that many experimental measurements do not correspond to a fundamental mode solution. For example, for a sub-critical system it is quite typical to experimentally measure multiplication, $\mathrm{K}$, etc., by placing a constant (time independent) source inside the system and directly measuring multiplication. In this case, we measure the entire fission chain starting from the source, without the equivalent of any settle time or cycles; in other words we are defining a non-fundamental mode result. In a fundamental mode solution the results are independent of the initial source; increasing entropy results in a loss of memory of the initial source distribution as the system settles into its fundamental mode. In a non-fundamental mode solution, the results can be quite dependent on the source distribution. For example, in a small system, with a fast neutron source most of the source neutrons can directly leak from the system without interacting (because the high energy neutron cross sections are so small), resulting in little multiplication of the source strength. In contrast, a thermal neutron source placed at exactly the same position can result in high multiplication (because the slow neutron cross section are so much bigger). This is but one simple example of how the same system with a fast or thermal neutron source can be measured, or calculated, to have very different neutron multiplication.

Most of the below discussion refers only to a fundamental mode solution, where the time variation is defined by a simple, single exponential time dependence. But this isn't a necessary limitation on these methods. For example, the dynamic criticality method described in this paper can be used to simulate non-fundamental modes by using no settle cycles, e.g., simulating the experiment described above using a fast or thermal neutron source can be easily done using the methods described below. Of course, for sub-critical systems you always have the option of running a source, rather than a criticality problem. The reader should be aware of this when using neutron transport codes, and define code input as appropriate to what you want to simulate, e.g., source or criticality problem, fundamental mode, with settle time, or non-fundamental mode, without settle time.

\section{Biased versus Unbiased Methods}

Since with today's large computers we can now use a true analog calculation to define the time dependence of a system (at least for some finite period of time), we are in a position to determine whether or not the below described criticality methods produce the same results as a true analog calculations, which we assume are correct. The question 
comes down to, when the system relaxes into its fundamental mode, and the true analog solution becomes separable in (space, energy, direction) and (time),

$$
N(r, E, \Omega, t)=N_{0}(r, E, \Omega) * \operatorname{Exp}[\alpha * t]
$$

Do the below described methods produce the same, correct spectrum $N_{0}(r, E, \Omega)$ and time dependence $\operatorname{Exp}\left[\alpha^{*} t\right]$ ? If a method produces the same results as the true analog method, we refer to the method as an unbiased result. However, if the method does not produce the same results we refer to this as a biased results. We are be interested in determining the source of any bias, so that we can predict when these methods will be accurate or inaccurate.

\section{The Right Answer for the Wrong Problem}

Some readers of preliminary versions of this paper objected to our saying that the K static method of criticality calculations, the method we were all taught in school, and that has been used for criticality calculations for over fifty years, can give incorrect answers. Their claim is that the K static method accurately solves the problem IT defines, which is true. However, except for systems that are close to critical $(K \sim 1)$ the $K$ static method cannot solve the problem WE define. We us, this is like being in a contest where you are asked to spell Czechoslovakia, and your answer is "Cat, C-A-T, Cat" - this is the right answer to the question IT defined (how to spell CAT), but it is not the answer to the question YOU were asked; to us this is an incorrect answer.

This is exactly what the K static method does, i.e., it doesn't answer the question WE ask. For example, if WE ask the question: what's K-eff for OUR system? The K static method answer is: if you reduce $\langle v\rangle$ by a factor of 1.58 this system will become critical. Maybe this is an interesting answer, but it isn't the answer to the question WE asked. The K static method assumes that if it has to modify the system reducing $\langle v\rangle$ by a factor of 1.58 to make this system critical, then K-eff of the OUR unmodified system must be 1.58. Sorry, but this simply is not true, as we will see below.

Let's assume YOU are interested in a system that is far from critical. Being not critical means that the system YOU are interested in is truly time dependent. Sorry, but the K static method cannot solve YOUR problem, since it can only solve static, time independent systems, which means in principle it can only solve system that are exactly critical. If you use the $\mathrm{K}$ static method to try and solve YOUR truly time dependent problem, it does not even attempt to solve your problem. Instead IT defines a different problem: a static, time independent problem that it can solve, and gives you only the solution to this time independent problem, without any indication that this may not be the correct answer to the problem YOU are interested in.

You the reader can decide for yourself whether or not you would be satisfied to be given the right answer to the wrong problem. To us giving the right answer for the wrong problem is not only incorrect, it is dangerous, if you are led to believe that the answer is correct for your problem. So Caveat Emptor (let the user beware) if you use the K static 
method, unless the system YOU are interested is close to critical, $\mathrm{K} \sim 1$. To see how much of a quantitative difference a true dynamic calculation versus a $\mathrm{K}$ static calculation can make, see the below example problems, particularly problem \#3.

In pointing out that the $\mathrm{K}$ static method can give incorrect answers if used in problems where the method does not imply, we are not totally rejecting the use of the $\mathrm{K}$ static method. When used for systems that are close to critical it remains the most efficient method of calculation, and its answers are correct, or at least correct enough; this is where the K static method applies. Proper use the K static method for more than the last fifty years has proven how effective the method can be. Our WARNING only applies to systems that are far from critical. Specifically, our warning is that the K static method simply doesn't apply to such systems, so that you should not be surprised if you misuse the method and obtain incorrect and misleading answers.

\section{Combing}

In discussing criticality methods we will use the expression neutron combing. Combing refers to population control, used in criticality calculations, because our computers have only a finite size (for a super-critical system), or to prevent our neutron population from decreasing to zero (for a sub-critical system).

For example, for a super-critical system, if we run a real analog calculation over some time interval the neutron population grows and could quickly exceed the size of our computer if we did not take some action. In this case if we start the time interval with 10,000 neutrons and 15,000 neutrons reach the end of time interval, combing refers to the neutron population control process we use to allow us to start the next time interval with the same total of 10,000 neutrons that we started the previous time interval, by discarding 5,000 of the 15,000 neutrons. This is equivalent to time dependent Russian roulette, to kill off excess neutrons without biasing the neutron population. 10,000 versus 15,000 neutrons may not sound like much of a problem, until you consider that this is a $50 \%$ increase in one time interval and we may run thousands of time intervals.

Similarly for a sub-critical system, if we run a real analog calculation over some time interval the neutron population decreases and could quickly go to zero if we did not take some action. In this case, if we start the time interval with 10,000 neutrons and 5,000 neutrons reach the end of the time interval, combing refers to the neutron population control process we use to allow us to start the next time interval with the same total of 10,000 neutrons that we started the previous time interval with, by statistically sampling each of 5,000 neutrons twice. This is equivalent to time dependent neutron splitting, to increase the neutron population without biasing the neutron population. 10,000 versus 5,000 neutrons may not sound like much of a problem, until you consider that this is a 50 $\%$ decrease in one time interval and we may run thousands of time intervals.

In summary: in the following discussion the expression combing refers to neutron population control, using importance sampling methods, which can be used as needed to either decrease the neutron population, using Russian roulette, or increase the neutron 
population, using neutron splitting. In particular we stress that the reader should not confuse neutrons that disappear or are created during combing with actual neutron absorption or production; these terms are only effects introduced by importance sampling to control the neutron population. As we will see below, how this is done in the various methods determines whether or not these methods are unbiased (accurate) or biased (inaccurate).

\section{Time independent and Time dependent: Sub-critical and super-critical}

Based on the model described above, for a super-critical system there is only a time dependent solution for the flux; there is no time independent, steady state solution. This means we can only have time dependent sources, e.g., a pulse of neutrons at time zero, or at some series of times, and we then follow the resulting time evolution of the flux. In contrast for a sub-critical system we can have either a fixed, time independent source, for which we can determine a time independent flux, or we can have a pulsed source, for which we can determine a time dependent flux.

Below we will be dealing with time dependent systems and examining methods of controlling the exponential changes in the neutron flux with time in such systems. The emphasis will be on super-critical systems, since in principle no such methods are required for sub-critical systems. For a sub-critical system we can use a fixed, time independent source, and solve for the multiplication, reactivity, removal time, etc., by solving such problems as source, rather than criticality problems.

Our ability to solve sub-critical problems as either source or criticality problems, using either a fixed, time independent source, or a pulsed, time dependent source, gives us an excellent means of checking the consistency of our methods.

\section{Neutron flux and neutron density and the fundamental mode}

A good deal of confusion can be avoided by properly interpreting the time derivative term in equation [1], and by initially writing the equation in terms of neutron density, rather than flux. The variable $N(R, \Omega, E, t)$ in this equation is the neutron flux, which is merely the product of the neutron speed (speed, not velocity) and the neutron density $v^{*} n(R, \Omega, E, t)$, so that the simplest interpretation of the time derivative term is the rate of change of the neutron density,

$$
\frac{1}{v} \frac{\partial N}{\partial t}=\frac{\partial n}{\partial t}
$$

If the equation is written in terms of neutron density, rather than flux, the only difference is that there is no $1 / v$ factor on the time derivative,

$$
\frac{\partial n}{\partial t}+\Omega * \nabla\left(v^{*} n\right)+\Sigma t^{*}\left(v^{*} n\right)=\iint[<v>* \Sigma f+\Sigma s] F\left(E^{\prime}, \Omega^{\prime}->E, \Omega\right)\left(v^{*} n\right) d E^{\prime} d \Omega^{\prime}+S
$$


In this form, it is easier to interpret the fundamental mode and the alpha static method described below. In the fundamental mode we assume that after some time period we have separation of the neutron density by (space, energy, direction) and (time), such that at all subsequent times, the variation of the neutron density can be described as,

$n(R, \Omega, E, t)=n(R, \Omega, E) * \operatorname{Exp}\left[\alpha^{*} t\right]$

In the dynamic method we allow the neutron population to grow over a period of time and, only at the end of the period of time, we uniformly comb the neutron population. Using the above form for the fundamental mode, we comb the neutron population uniformly to return it to the neutron population that we started with at the beginning of the time step; we will then start the same number of neutrons in our next time step. Please remember this: we uniformly comb neutron population, not neutron flux or anything else.

Letting the neutron population grow or decrease may not be the most efficient way to solve this problem, e.g., as we let the population grow we must transport more neutrons and the running time of the problem grows. Rather than waiting until the end of the time interval, it may be more efficient to comb the neutrons throughout the time interval to maintain the neutron population as constant. That's all the alpha static method does. In the alpha static method we assume that the system is in its fundamental mode, so that,

$$
\frac{\partial n}{\partial t}=\alpha^{*} n
$$

If we take no action, this says that during the time interval the neutron population will change at a rate of $\alpha^{*} n$. Therefore, to control the neutron population and keep it constant, throughout the time step we will comb the population by just this factor, in opposition to the natural growth or decrease of the neutron population. This means in our equation for the neutron population, we replace the time derivative $\frac{\partial n}{\partial t}$ by $\alpha^{*} n$,

$$
\alpha^{*} n+\Omega * \nabla\left(v^{*} n\right)+\Sigma t^{*}\left(v^{*} n\right)=\iint\left[<v>{ }^{*} \Sigma f+\Sigma s\right] F\left(E^{\prime}, \Omega^{\prime}->E, \Omega\right)\left(v^{*} n\right) d E^{\prime} d \Omega^{\prime}+S
$$

Re-arranging terms,

Super-critical $(\alpha>0)$,

$$
\Omega^{*} \nabla\left(v^{*} n\right)+\Sigma t^{*}\left(v^{*} n\right)+\alpha^{*} n=\iint\left[<v>{ }^{*} \Sigma f+\Sigma s\right] F\left(E^{\prime}, \Omega^{\prime}->E, \Omega\right)\left(v^{*} n\right) d E^{\prime} d \Omega^{\prime}+S
$$

Sub-critical $(\alpha<0)$,

$$
\Omega * \nabla\left(v^{*} n\right)+\Sigma t^{*}\left(v^{*} n\right)=-\alpha^{*} n+\iint[<v>* \Sigma f+\Sigma s] F\left(E^{\prime}, \Omega^{\prime}->E, \Omega\right)\left(v^{*} n\right) d E^{\prime} d \Omega^{\prime}+S
$$


Note that in the above equation for $\alpha<0$, the term $-\alpha^{*} n$ is positive. Please focus on our objective, which is to uniformly comb the neutron population. In order to accomplish this, we add to our equation a term that mathematically looks like either absorption $(\alpha>0)$ or production $(\alpha<0)$, at exactly the rate necessary to counteract the natural growth of the neutron population. But keep in mind that this is merely a mathematical trick; physically we are not saying that these terms cause actual absorption or production of neutrons; we are merely using these terms for importance sampling to maintain the neutron population.

In the above equation for neutron population, it is easy to see that we are uniformly combing the neutron population, i.e., the term $\alpha$ in $\alpha^{*} n$ has no dependence on (space, energy, direction), so if we were solving this equation, people wouldn't be so confused about the alpha static method.

Unfortunately, neutrons alone do not produce a physical observable; we also have to consider their motion. That's why the equation we actually solve uses neutron flux, rather than neutron population. Naturally it is trivial to convert the above equations from neutron population to neutron flux; indeed all of the terms in these equation except for $\alpha^{*} n$ already involve flux. Therefore, to complete the transformation from neutron density to flux, we need merely multiply and divide this term by neutron speed $(v)$,

Super-critical $(\alpha>0)$,

$\Omega^{*} \nabla N+\Sigma t^{*} N+\frac{\alpha}{v} * N=\iint[<v>* \Sigma f+\Sigma s] F\left(E^{\prime}, \Omega^{\prime}->E, \Omega\right) N d E^{\prime} d \Omega^{\prime}+S$

Sub-critical $(\alpha<0)$,

$\Omega^{*} \nabla N+\Sigma t^{*} N=-\frac{\alpha}{v} * N+\iint[<v>* \Sigma f+\Sigma s] F\left(E^{\prime}, \Omega^{\prime}->E, \Omega\right) N d E^{\prime} d \Omega^{\prime}+S$

This form appears to bias the absorption in velocity space until one realizes that the $\alpha / v$ term is multiplied by a flux, not a neutron density. Uniform combing of the population density by a factor $\alpha$ means combing of the flux by a factor $\alpha / v$; that's where this rather strange looking term comes from.

Since this is an important concept, let us state it in another form. When the variable in our equation is flux we mathematically add a term that looks like reactions [ $\left.\Sigma x * N=\Sigma x *\left(v^{*} n\right)\right]$ to allow us to uniformly comb the neutron population. If we want to uniformly comb neutron population, for this term we need the same reaction rate at all energies, which means $\Sigma x^{*}\left(v^{*} n\right)=\alpha^{*} n$. Obviously this means our mathematical reaction cross section must be $\Sigma x=\alpha / v$. Again, focus on the point that this is merely a mathematical concept to allow us to control the neutron population. 
See the appendix for a discussion of time absorption and production.

Bottom line: The alpha static flux equation does not include an additional term corresponding to physically real absorption $(\alpha>0)$ or production $(\alpha<0)$. These terms have been added to our equation as a mathematical trick, which is equivalent to time dependent importance sampling, using neutron splitting or Russian roulette. Most important to understand is that inclusion of these terms does not bias the flux distribution or prevent it from achieving its fundamental mode shape, and it is completely equivalent to what we do in a dynamic criticality calculation. The only difference between the dynamic and alpha static methods is that the dynamic method does not comb the neutron population until the end of a time interval, whereas the alpha static method combs neutron production continuously throughout a time interval. And most important: both methods get exactly the same answer.

Let us point out that most textbooks do not seem to understand this $\alpha / v$ method at all,

1) They say this method cannot be valid because of the inclusion of this nonphysical $\alpha / v$. These books do not seem to understand the basic idea of what we are trying to accomplish using importance sampling to achieve neutron population control.

2) They say that this method cannot be used for sub-critical system because for negative $\alpha$ the total cross section can become negative. Here they really demonstrate that they do not understand that to correctly comb for super-critical systems $(\alpha>0)$ we must remove neutrons, hence the time absorption, but for sub-critical systems $(\alpha<1)$ we must add neutrons, hence the time production. We always deal with positive probabilities, and we never subtract a term from the total cross section.

\section{The True Analog solution}

We will start from our Boltzmann equation and without making any additional approximations we will derive the time dependent fundamental mode solution that we expect for a true analog criticality calculation. To see this behavior we return to equation [1], in this case without the source term, $S$ (since we are only interested in the fundamental mode solution), multiply by neutron speed $(v)$, and integrate over space, energy, and direction, to obtain the equation

$$
\frac{d N_{0}}{d t}+v\left[L^{*} N_{0}+\Sigma t * N_{0}\right]=v[<v>* \Sigma f+\Sigma s] N_{0}
$$

Here $N_{0}(t)$ is the system integral scalar flux which is now only a function of time $(t), \mathrm{L}$ represents leakage, and the others items are all system average integral quantities. Subtract the scattering cross term $\left(\Sigma s^{*} N_{0}\right)$ from both sides of the equation (scatter does not directly effect the neutron population; it only indirectly effects criticality through its effect on the moderation and leakage of the neutrons). 
$\frac{d N_{0}}{d t}+v[L+\Sigma a]^{*} N_{0}=v[<v>* \Sigma f] N_{0}$

Here the absorption cross section $(\Sigma a)$ includes fission $(\Sigma f)$. Collecting all terms together,

$\frac{d N_{0}}{d t}=v[<v>* \Sigma f-(L+\Sigma a)] N_{0}$

Here we can already see the simple exponential solution. Rearranging terms,

$\frac{d N_{0}}{d t}=v(L+\Sigma a)[<v>* \Sigma f /(L+\Sigma a)-1] N_{0}$

Here we will define,

$K=<v>* \Sigma f /(L+\Sigma a) \quad$ the effective neutron multiplication factor

$T_{R}=1 /[v(L+\Sigma a)] \quad$ the removal time

$\frac{d N_{0}}{d t}=\left[(K-1) / T_{R}\right] N_{0}$

$\frac{1}{N_{0}} \frac{d N_{0}}{d t}=\frac{d \operatorname{Ln}\left[N_{0}\right]}{d t}=\left[(K-1) / T_{R}\right]$

Since we assume that the properties of the medium are time independent, the solution is,

$N_{0}(t)=N_{0}(0) \operatorname{Exp}\left(\alpha^{*} t\right)$

where,

$\alpha=\left[(K-1) / T_{R}\right]$

For example, for a non-multiplying system $(\mathrm{K}=0)$, we have

$\alpha=-1 / T_{R}=-v^{*}[L+\Sigma a]=$ the neutron removal rate

Here we can see that in the absence of multiplication $(\mathrm{K}=0)$, the time constant, $\alpha$, is defined solely by the rate of removal of neutrons from the system. In general $\alpha$ is defined by the balance between production, absorption, and leakage rates,

$\alpha=v[<v>* \Sigma f-(L+\Sigma a)]=$ production rate - (leakage and absorption rates) 
What is described above is exactly how criticality calculations are performed in any type of neutron transport calculation, be it Monte Carlo or deterministic. For the system as a whole determine the number of neutrons produced, absorbed and leaked, these define $\mathrm{K}$, and the rate at which neutrons are removed, which defines the removal time $T_{R}$, the combination of $\mathrm{K}$ and removal time $T_{R}$ then uniquely define the system time constant $\alpha$.

In a true analog calculation we can start from any instantaneous source distribution [that defines the initial condition $N_{0}(0)$ ] and follow the evolution of the flux in space, energy, direction, and time. We expect that after a sufficiently long period of time the system will relax into its fundamental mode, and at all subsequent times the flux at all space, energy, and directions, will have exactly the same common time dependence. If we had infinite computer resources, we could perform the calculation exactly as described here. Unfortunately, even today's large computers are not adequate to perform calculations exactly as described here, e.g., with the simple instantaneous model we are using, for a highly super-critical system, the flux can theoretically increase by $10^{100}$ in microseconds. Therefore we introduce certain models that allow us to perform the calculations with resources that we have today. We will divide these into dynamic and static criticality models.

As we will see below, in a dynamic criticality calculation, we can independently calculate each of the three terms: $\alpha, K$ and $T_{R}$. In a static ( $\mathrm{K}$ and $\alpha$ ) criticality calculation, we can only calculate $K$ and $T_{R}$, but from the above equation we can then uniquely define $\alpha$. In principle, for consistency between static and dynamic calculations, we need merely ensure that in both cases we use exactly the same definitions for $K$ and $T_{R}$ (see the appendix for the unique definitions used here).

\section{Methods of Solution}

\section{Dynamic Criticality}

In principle, in a Monte Carlo calculation, we can start from any arbitrary source and follow the time evolution of the neutron flux until it has relaxed into its fundamental mode and we have the expected simple, single exponential variation of the flux, and that's it: we have solved the problem - this is what we described above as the true analog solution.

In reality, the exponential variation of the flux can rapidly cause the number of neutrons being tracked to exceed the memory of a computer (if the system is super-critical, $\alpha>0$ ), or cause the number of neutrons to approach zero, so that there is nothing left to track (if the system is sub-critical, $\alpha<1$ ). These problems are easily overcome by tracking neutrons for some time interval and then using neutron population control to comb the neutron population, to statistically return the neutron population to its size at the beginning of the time interval. By randomly selecting from those neutrons that reach the end of the time interval, we can define an unbiased sample of the neutron population that we can use to continue our calculation into the next time interval. This has been the historical approach to solving a dynamic criticality problem. 
Today's gigabyte sized computers are large enough that we can actually track neutron population changes of millions or even billions, which gives us an independent means of insuring that our traditional approach to solving dynamic criticality problems using neutron population control agrees with the results allowing "unlimited" population increase or decrease. This agreement has now been verified by using TART [1] to solve systems as source problems where we allow unlimited growth (over some finite time interval) and as criticality problems where we use population control. Therefore we can be sure that our traditional method of solving dynamic criticality problems is an unbiased method that produces accurate results.

Since this is an important concept for this paper, let us again describe the dynamic method with combing. At the beginning of a time step, we start with a given number of neutrons, $n 0(0)$. By the end of the time step, the neutron population will have changed to $n 0(t)=n 0(0) \operatorname{Exp}\left(\alpha^{*} t\right)$. We wish to comb the population back to what it was at the beginning of the time step. Obviously, in order to do this we must comb the population by a factor of $\operatorname{Exp}\left(\alpha^{*} t\right)$, i.e., for a sub-critical system $(\alpha<0)$, we must comb to increase the population, and for a super-critical system $(\alpha>0)$, we must comb to decrease population. For example, if at the end of a time step we have twice as many neutrons as we had at the beginning of the time step, we will use Russian roulette to discard half of them, and thereby start the next time step with the same number of neutrons that we had at the beginning of the previous time step. As a second example, if at the end of a time step we have half as many particles as we had at the beginning of the time step, we will use particle splitting to use each particle twice, and thereby start the next time step with the same number of particles that we had at the beginning of the previous time step. The important point to note is that by assuming that the system is in its fundamental mode, we have a separation of the flux into (space, energy, direction) and (time), such that at all space, energy and directions, the time dependence is exactly the same, $n 0(t)=n 0(0) \operatorname{Exp}\left(\alpha^{*} t\right)$, which means that for population control in time, we must comb the neutron population uniformly with equal probability.

\section{Static Methods}

There are several different methods that textbooks describe as "changing" the actual time dependent variation of a system. What the textbook description of all these methods have in common is that they introduce a parameter to make the system appear to be critical, and therefore time independent, or static. The two methods that we will discuss here both start from the definition of the effective neutron multiplication factor that we defined above

$$
K=<v>* \Sigma f /(L+\Sigma a)=\text { Production/[Leakage }+ \text { Absorption }]
$$

In the so called $\mathbf{K}$ static method we vary the production of neutrons to make the system critical. In the so called alpha static method we vary the absorption $(\alpha>0)$ or

production $(\alpha<0)$ of neutrons to make the system critical. The advantage of these 
methods is that at least historically they were considered to be much easier methods to use, compared to solving a dynamic criticality problem, and for a system close to critical these static methods yield fairly accurate answers. Unfortunately, the fact that these different methods yield similar results for systems near critical has led people to believe that this is always true. We will show below that this isn't true for systems that are far from critical, and that the differences can be large and important. First let us give you the standard textbook description of these static methods.

\section{K Static Criticality}

We start from equation [1],

$$
\frac{1}{v} \frac{\partial N}{\partial t}+\Omega^{*} \nabla N+\Sigma t^{*} N=\iint[<v>* \Sigma f+\Sigma s] F\left(E^{\prime}, \Omega^{\prime}->E, \Omega\right) N d E^{\prime} d \Omega^{\prime}+S
$$

and introduce a parameter $\mathrm{K}$ modifying neutron production to make the system time independent, $\frac{1}{v} \frac{\partial N}{\partial t}=0$, i.e., critical,

$\Omega^{*} \nabla N+\Sigma t^{*} N=\iint\left[\frac{<v>}{K} * \Sigma f+\Sigma s\right] F\left(E^{\prime}, \Omega^{\prime}->E, \Omega\right) N d E^{\prime} d \Omega^{\prime}$

Starting from an arbitrary source distribution, we iterate this equation, neutron generation by generation, to determine the value of $\mathrm{K}$ that will make this system critical. Note, that this is an exact balance equation ONLY if we know the correct value of $K$ to use. But during iteration while to are trying to define $K$, this is not a true balance equation.

Let us restate the question posed by the K static method: for any given system, how much do we have to change $\langle v\rangle$ in order to make the system critical, and what is the flux distribution for this modified system? This is an interesting question, but it is not the question we want answered; we want to know what is the flux in the original, unmodified system. As we will see below, by posing the question in this form, the $\mathrm{K}$ static method is not solving the problem we are interested in, and generally, except for a system close to critical, there is no simple relationship between the modified exactly critical problem solved by the K static method, and the non-critical problem that we are interested in.

\section{Alpha Static Criticality}

Again we start from equation [1], but in this case we assume exponential variation of the flux with time,

$$
\frac{\partial N}{\partial t}=\alpha^{*} N
$$


which gives us the equation,

$$
\frac{\alpha}{v} N+\Omega^{*} \nabla N+\Sigma t^{*} N=\iint[<v>* \Sigma f+\Sigma s] F\left(E^{\prime}, \Omega^{\prime}->E, \Omega\right) N d E^{\prime} d \Omega^{\prime}
$$

For a super-critical system $(\alpha>0)$ the lead term is combined with the cross section term to give us the $\frac{\alpha}{v}$ time absorption equation,

$\Omega * \nabla N+\left[\Sigma t+\frac{\alpha}{v}\right]^{*} N=\iint[<v>* \Sigma f+\Sigma s] F\left(E^{\prime}, \Omega^{\prime}->E, \Omega\right) N d E^{\prime} d \Omega^{\prime}$

For positive $\alpha$ adding this term to the total cross section is mathematically equivalent to an additional $1 / \mathrm{v}$ absorption uniformly distributed throughout the entire system.

For a sub-critical system $(\alpha<0)$ we move the lead term to the right hand side of this equation to give us the $\frac{\alpha}{v}$ time production equation,

$\Omega^{*} \nabla N+\Sigma t^{*} N=-\frac{\alpha}{v} N+\iint[<v>* \Sigma f+\Sigma s] F\left(E^{\prime}, \Omega^{\prime}->E, \Omega\right) N d E^{\prime} d \Omega^{\prime}$

For negative $\alpha$ (making $-\frac{\alpha}{v} N$ positive) adding this term to the right hand side is mathematically equivalent to an additional $1 / \mathrm{v}$ production uniformly distributed throughout the entire system.

Again, please do not become confused by this $\alpha N / v$ term; it MUST be considered only a mathematical trick introduced for neutron population control, and MUST NEVER be interpreted as actual physical absorption or production in the system.

Starting from an arbitrary source distribution we iterate this equation, neutron generation by generation, to determine the value of $\alpha$ that will make this system critical. Note, that this is an exact balance equation ONLY if we know the correct value of $\alpha$ to use. But during iteration while to are trying to define $\alpha$, this is not a true balance equation.

\section{Time versus generations}

The dynamic method tracks neutrons in time. As defined in textbooks, the alpha and $\mathrm{K}$ static methods make a system time independent and they track neutrons generation by generation. There can be important differences between these two approaches. For example, consider a sphere filled with only water, so that there is no neutron production. If we pulsed the sphere of water we expect it to eventually relax into its fundamental 
mode, and thereafter we will see an exponential decrease in the flux. That's what has physically been observed in any number of actual experimental measurements. In this case the dynamic method would have no problem at all determining that for this system $\mathrm{K}=0$, and it would also define the neutron removal time and system time constant $(\alpha)$. In contrast, because there is no neutron production, the $\mathrm{K}$ static solution would end after one generation, and it wouldn't be able to tell us anything except that $\mathrm{K}=0$. Note, that in this case the actual time dependent leakage from this sphere of water exponentially decreases and at least in principle this distribution extends on to infinite time. By tracking all neutrons through one generation the $\mathrm{K}$ static method has at least in principle tracked all neutrons for their entire lifetime, and extended its calculation out to infinite time - not just through one average neutron removal time, as we might expect if generations are somehow closely related to time. Even though there are obvious differences between time and generations, the normal formulation of the static methods ignore these differences, seemingly completely ignoring time, and iterates generation by generation.

\section{Time Dependent versus Time Independent}

As it states in the introduction, this paper focuses on systems that are far from critical. Therefore we must focus on the fact that the real system that we are interested in is truly time dependent. Obviously the dynamic method includes this effect, by solving for results as a function of time. Less obvious is that the alpha static method also includes the effect of time through the inclusion of the term $\alpha^{*} N / v$, which corresponds to either time absorption $(\alpha>0)$ or time production $(\alpha<0)$; see the appendix for details of time absorption and production. In contrast, the $\mathrm{K}$ static method does not include the effect of time, and this contributes to the bias that this method can introduce. With the $\mathrm{K}$ static method we are not solving for the time dependent system that we are interested in. We are solving for a modified system where $\mathrm{K}$ has been used to artificially modify the production to make the system critical. If the system were truly critical then there wouldn't be any time dependent effects to consider, and the $\mathrm{K}$ static method would produce accurate answers, as we know from experience it does. Unfortunately, we really aren't interested in the modified, exactly critical system modeled by the K static system; we are only interested in the original, time dependent system, modeled by the dynamic method. The difference between these models we think can best to seen by casting all of these methods in a time form.

\section{All Time Dependent Approaches}

I (one author, DEC) never liked the above textbook description of these "static" methods, where they are described as making the system time independent, and instead of using time we track neutrons generation by generation. Consider that we iterate to define $\mathrm{K}$ and alpha, which means when we finish the calculation the system is time independent, but throughout our iterations the system is time dependent, since we have not yet defined the correct $\mathrm{K}$ or alpha to make it critical (only a critical system is exactly time indepemdent). Therefore rather than simply immediately assuming that the static methods make the system time independent, $\partial N / \partial t=0$, we can assume that this term is still present in our balance equation, and as we iterate to converge on the correct value of $\mathrm{K}$ or $\alpha$, this will 
"naturally" cause the time dependence to approach zero, i.e., that's what $\mathrm{K}$ and $\alpha$ are designed to accomplish.

We think it is easier to "see" the effects introduced by these methods if we describe them in a time dependent form. We will compare the three different methods all solved as time dependent equations. What we will show is that the three methods differ only in how they comb the neutron distribution to control population. There is no "phony" absorption or production to really make the systems time independent; all there is, is importance sampling to control population.

In all three cases we will solve the time dependent form of our equation [1].

$$
\frac{1}{v} \frac{\partial N}{\partial t}+\Omega^{*} \nabla N+\Sigma t^{*} N=\iint[<v>* \Sigma f+\Sigma s] F\left(E^{\prime}, \Omega^{\prime}->E, \Omega\right) N d E^{\prime} d \Omega^{\prime}+S
$$

In order to deal with the rate of change of flux or neutron population, we multiply by the neutron speed $(v)$,

\section{Dynamic method}

$$
\frac{\partial N}{\partial t}+v\left\{\Omega^{*} \nabla N+\Sigma t^{*} N\right\}=v \iint[<v>* \Sigma f+\Sigma s] F\left(E^{\prime}, \Omega^{\prime}->E, \Omega\right) N d E^{\prime} d \Omega^{\prime}+v^{*} S
$$

The first method is the dynamic criticality calculation where we solve this equation without any changes: we will track neutrons over a time interval, and comb all neutrons only at the end of the time interval to maintain population control. We maintain population by combing to decrease or increase the population of neutrons that reach the end of the time step by a factor $\operatorname{Exp}\left(\alpha^{*} t\right)$, independent of the energy, position, or direction of the neutron when it reached the end of the time step. Because we are doing this combing over a fixed time interval, what we are uniformly combing is the rate of change of the neutron population. In order to converge to the correct fundamental mode solution we must iterate over a number of time intervals to determine the correct stationary flux and simple, exponential time dependence. For the dynamic method what we describe here is essentially only repeating the method as described above.

\section{K Static method}

$$
\frac{\partial N}{\partial t}+v\left\{\Omega * \nabla N+\Sigma t^{*} N\right\}=v \iint\left[\frac{<v>}{K} * \Sigma f+\Sigma s\right] F\left(E^{\prime}, \Omega^{\prime}->E, \Omega\right) N d E^{\prime} d \Omega^{\prime}+v^{*} S
$$

The second method is the K static method. Again we will track neutrons over a time interval, but in this case we will comb the rate of neutron production throughout the time interval, by defining our parameter $\mathrm{K}$, such that the neutron population at the end of 
the time interval is the same as at the start of the time interval, i.e., $\frac{\partial N}{\partial t}=0$. In order to converge to the desired time independent behavior, we calculate over a series of time intervals, and during these time intervals the flux is actually time dependent, since as yet we have not determined the required K; that's one reason that to us it makes more sense to look at this method in a time dependent form.

\section{Alpha Static method}

Super-critical $(\alpha>0)$

$$
\frac{\partial N}{\partial t}+v\left\{\Omega * \nabla N+\Sigma t^{*} N\right\}+\alpha N=v \iint[<v>* \Sigma f+\Sigma s] F\left(E^{\prime}, \Omega^{\prime}->E, \Omega\right) N d E^{\prime} d \Omega^{\prime}+v^{*} S
$$

Sub-critical $(\alpha<0)$

$$
\frac{\partial N}{\partial t}+v\left\{\Omega * \nabla N+\Sigma t^{*} N\right\}=-\alpha N+v \iint[<v>* \Sigma f+\Sigma s] F\left(E^{\prime}, \Omega^{\prime}->E, \Omega\right) N d E^{\prime} d \Omega^{\prime}+v^{*} S
$$

The third method is the alpha static method. Again we will track neutrons over a time interval, but in this case we will comb the rate of neutron absorption or production throughout the time interval, such that the neutron population at the end of the time interval is the same as at the start of the time interval, i.e., $\frac{\partial N}{\partial t}=0$. In order to converge to the desired time independent behavior we calculate over a series of time intervals, and during these time intervals the flux is actually time dependent, since as yet we have not yet determined the required alpha $(\alpha)$; that's one reason that to us it makes more sense to look at this method in a time dependent form.

\section{Time versus Generation}

This time dependent formulation of the two static methods described above is exactly equivalent to the methods used to solve the time independent static methods. The only difference in the approaches is that the traditional static methods solve the equations generation by generation, whereas here we solve them time step by time step. Neutrons do not move through matter in lockstep generation by generation; they actually move in time. Stated in their time dependent form we can see that the only difference between the three methods is how they comb the neutrons to maintain population control.

\section{Importance Sampling: Population Control}

Let us again stress the point that all of these methods use combing ONLY because they need population control. The dynamic method isn't saying that instantaneously, at the end of each time interval, the neutron population really changes to agree with what it was at 
the beginning of the time interval. Neither is the $\mathrm{K}$ static method saying that the production of neutrons throughout the time is really $\frac{\langle v\rangle}{K}$ rather than $\langle v\rangle$. Nor is the alpha static method saying that there really is some additional absorption or production $\alpha^{*} N / v$ throughout the time interval to insure that the neutron population at the end of the time interval is the same as at the end of the time interval.

What they are all doing is importance sampling to control population, and the only important question is: is their importance sampling biased or unbiased?

The dynamic method, by combing the rate of change of the neutron population only at the end of time steps, uniformly in energy, position, and direction by the factor by which the population changed during the time interval, $\operatorname{Exp}\left(\alpha^{*} t\right)$, gives an unbiased sample of the flux to continue the calculation from one time step to the next. As such this method produces accurate results. Again, we will mention that today our computers are large enough to allow us to verify that this method of combing gives exactly the same answers as a true analog calculation, where we allow the neutron population to change without combing, i.e., this combing is unbiased and correctly simulates true time dependent variation.

The K static method, by combing neutron production throughout the time interval, biases the flux in energy, e.g., for a super-critical system it reduces the high energy neutron production relative to other neutrons, which results in a shift of the neutron spectrum toward lower energies. For heterogeneous systems it also biases the flux in space. Obviously neutron production by fission can only occur in fissile material, so that for a super-critical system by reducing neutron production, relative to the neutrons transporting outside of the fissile material, it results in a shift of the neutron flux from fissile to non-fissile materials. We will see both of these biasing effects in the example problem results presented below. Note that for an exactly critical system the neutron population remains constant with time, so that there is no combing, and therefore no bias introduced by the $\mathrm{K}$ static method; which explains why the $\mathrm{K}$ static method is accurate for critical and even near critical systems.

The alpha static method, if we think in terms of numbers of events, in combing absorption or production by a factor of $\alpha / v$ sounds as if the method biases the flux. However, for transport over a fixed time interval we must concentrate not on the number of events, but rather the rate of events. In terms of the rate of events from the above equation, we can easily see that the alpha static method combs the neutron population by a factor $v^{*}(\alpha / v)=\alpha$, exactly the same factor $(\alpha)$ used by the dynamic method, uniformly in space, energy and direction. We can see this from the term $\alpha^{*} n$ : for a super-critical system, this appears in the equation as a uniform "absorption" term to reduce the neutron population, and for a sub-critical system this appears as a uniform "production" term to increase the neutron population. Again, try not to think of this as "real" absorption or production; it is only a form of importance sampling for population control. 
In other words the alpha static method is doing exactly the same combing by $\operatorname{Exp}\left(\alpha^{*} t\right)$ as the dynamic method. The only difference between the dynamic and alpha static methods is that the dynamic method allows the population to change by a factor of $\operatorname{Exp}\left(\alpha^{*} t\right)$ throughout the time interval and then instantaneously combs the population at the end of the time interval. In contrast, the alpha static method combs the population continuously throughout the time interval to insure that the population reaching the end of the time interval is the same as the population at the beginning of the time interval. Otherwise the two methods are identical and most important for the discussion here, both methods produce the same accurate results.

This is an important point, so we will repeat: the dynamic and alpha static methods are equivalent for criticality calculations - but only for criticality calculations. The dynamic method can solve the general equation [1] without any further assumptions. The assumption by the alpha static method of a single, simple exponential variation of the flux limits this method to criticality calculations.

\section{Combing}

Some people do not like the alpha static method because of the "phony" absorption or production introduced by this method. However, if you think of this not as real absorption or production, but merely as time dependent importance sampling to control population growth you may have less trouble accepting this method. Let us say it again: the alpha static method is doing exactly the same thing as a dynamic calculation, combing neutrons to control population, and both produce accurate results. They are not really doing "phony" absorption or production; what they are actually doing is time dependent splitting or Russian roulette for population control.

If you still have trouble accepting this $\alpha / v$ combing used by the alpha static method uniformly throughout each time step, you should have even more trouble accepting the dynamic method where the population is allowed to exponentially change throughout each time step, and then instantaneously changes at the end of each time step. In fact both are easily understand if you understand the basic concept of using importance sampling for neutron population control.

\section{Convergence}

A Monte Carlo code will solve a criticality problem by starting from a given neutron source distribution, and first iterating through what we call settle cycles, to allow the neutron distribution to settle into its fundamental mode. Only after the settle cycles are completed do the codes start to really accumulate statistical information and test for convergence to the final answer. The total time to run a problem is therefore the time to run the settle cycles (complete overhead, in that the results do not directly affect the final answer), and the time after settle cycles until convergence. Naturally we are anxious to minimize the total running as long as we can maintain the accuracy of our solution. 
Important considerations in selecting one solution algorithm versus another include, does the method converge to the right answer, and how long does it take to converge to the right answer? One of the nice features of the $\mathrm{K}$ static method running generation by generation is that it rapidly converges, unfortunately, as we will see below unless the system is close to critical, it often converges to the wrong answer. Actually let us rephrase that: the $\mathrm{K}$ static method converges for the problem it posed (what change in $\langle v\rangle$ will make the system critical?), but the problem it posed is not the one we are interested in. In terms of the number of time steps to settle, the dynamic method can converge slowly, as can the alpha static method running generation by generation. This means that compared to the popular K static method, the other methods can require more settle cycles, and care must be used to avoid false convergence.

Also for the dynamic and alpha static methods care must be used to insure convergence for highly sub-critical systems. This problem can be understood by again examining the assumption of modes, and relaxation into the fundamental mode. We assume that starting from any initial source distribution, the time dependence of the system can be defined as,

$$
N(r, E, \Omega, t)=\sum_{j=0}^{\infty} N_{j}(r, E, \Omega) * \operatorname{Exp}\left[\alpha_{j}^{*} t\right]
$$

For a super-critical system we assume only one of the values of $\alpha$ is positive, so that with time this mode will increase and exponentially separate itself from all of the other decreasing modes. In contrast, for a sub-critical system, all of the values of $\alpha$ are negative, so that with time, all of the modes are decreasing, and for highly sub-critical systems, it may take a considerable period of time, before the fundamental mode separates sufficiently from the sum of all other modes.

For super-critical systems the distributions are combed to decrease the population by a factor $\operatorname{Exp}\left[-\alpha^{*} t\right]$, which tends to exponentially diminish with time the effect of the initial source distribution, which results in a stable situation. In contrast for sub-critical systems, the distributions are combed to increase the population by a factor $\operatorname{Exp}[+\alpha * t]$, which tends to exponentially increase with time the effect of the initial source distribution, which can result in an unstable situation. For such systems it may be better to run a true analog calculation, to avoid combing, and therefore eliminate this problem.

The net result is that care has to be used to guarantee accurate convergence for highly sub-critical systems. For super-critical system we are often blasé about defining the initial source distribution, since we assume the other modes will exponentially decrease with time. For a sub-critical system you can help speed convergence by trying to improve the initial source distribution to be closer to the fundamental mode distribution, thereby minimizing the magnitude of the other modes, in the above sum, and thereby allowing the fundamental mode to more rapidly separate itself from the sum of the other modes.

\section{Proof of the Pudding}


How much of an effect are we talking about? Let's see by calculating results for a few typical systems. To see the effects we are interested in, we need only consider a few simple systems, namely fast and slow neutrons, homogeneous and heterogeneous geometry. To illustrate that all three methods produce the same answer for critical systems, we will first consider a near critical system. For the following problems as extreme cases to test the various methods, we consider systems that are highly supercritical. For the last problem we consider a sub-critical system.

The important point to note is that if two methods produce exactly the same flux spectrum, all of the integral answers will be the same for both methods. Therefore we will concentrate on showing both the flux spectra (actually neutron production spectra) and integral results; the first helps to qualitatively explain why there are differences and the latter demonstrates quantitatively how large the differences are in integral parameters.

Below we provide a complete description of each problem, so that anyone else can independently reproduce these results. For consistency, all of the following problems use only prompt $\langle v\rangle$, i.e., delayed neutrons are completely ignored. For all problems we will define the composition in terms of atom, not gram, ratios, e.g., water is 2 atoms of hydrogen to 1 atom of oxygen, with its overall density adjusted to 1 gram/cc. All time dependent results are given in units of microseconds (usec), and neutron energy is in $\mathrm{MeV}$.

The neutron production spectra shown below correspond to,

$<v(E)>\Sigma f(E) N 0(E)$

with the integral over energy normalized to one neutron Actually this production spectrum is composed of all neutron production, fission, $(n, 2 n),(n, 3 n)$, etc.; the above notation only indicating fission, is used merely for simplicity. Note, that the neutron energy $(E)$ here is the energy at which the neutrons interacted to cause fission, not the energy of the spectrum of neutrons emitted by the fission. In this form we can easily see which energies are driving any given system by causing the production of fission neutrons, e.g., see the below figures.

\section{TART Results}

All of the following results were produced using the TART code [1]. TART has traditionally been able to perform dynamic and $\mathrm{K}$ static criticality calculations. For this paper TART was extended by adding an alpha static calculation. For all types of calculations (critical or source) TART calculates analog and expected results for all of the parameters of interest here; agreement between analog and expected results is an excellent indicator of convergence. Where possible, the following results were checked using a number of other neutron transport codes, including the Monte Carlo codes MCNP [2] and Mercury [3], and the deterministic $\mathrm{S}_{\mathrm{n}}$ code AMTRAN [4]. 


\section{Important Parameters}

In a time dependent calculation there is more of importance than merely defining the time constant, $\alpha$. A common mistake is to run a $\mathrm{K}$ static calculation to define $\mathrm{K}$ and the removal time, and to then assume this $\mathrm{K}$ and removal time are correct and to use them as input into a dynamic calculation (in particular, the removal time is used to define a dynamic time step). As we will see below this assumption can be very inaccurate, which can lead to errors in related quantities, such as fission rates, and in turn energy deposition.

For any type of problem (critical or source problem) TART [1] always calculates the analog and expected $\mathrm{K}$, removal time, and time constant, independently for each system and type of calculation, and these definitions are based on the real, time dependent systems, not the systems modified by $\mathrm{K}$ or $\alpha$ to make the system appear static (see, the appendix for details). For example, the results below include these parameters for each system, for each of the three different methods of solution, and you should note that the $\mathrm{K}$ static results can be quite different from the dynamic or alpha static results. In all cases the calculations were run for a sufficiently long time that the analog and expected results statistically converged to agree.

Problem \#1 - A homogeneous, fast neutron system, near criticality: We will start with just about the simplest possible system, namely Godiva, a bare sphere of uranium. There are actually many published specifications for Godiva. Here are the specifications that we used,

Spherical radius: $8.7407 \mathrm{~cm}$

Density: $\quad 18.7398$ grams/cc

Composition: $\quad 922350.937695$ atoms

$92238 \quad 0.052053$ atoms

922340.010252 atoms

normalized to 18.7398 grams/cc overall density

This is a good problem to start with because it is geometrically so simple and close to prompt critical, so we can use it to verify that all the methods we are comparing agree for a critical system

$\begin{array}{llll} & \text { Dynamic } & \text { K Static } & \text { Alpha Static } \\ \text { K-eff } & 0.9954 & 0.9958 & 0.9954 \\ \text { Alpha/usec } & -0.739 & -0.680 & -0.739 \\ \text { Removal Time usec } & 6.124 \mathrm{e}-3 & 6.110 \mathrm{e}-3 & 6.124 \mathrm{e}-3 \\ \text { Median Neutron Energy MeV } & 0.867 & 0.871 & 0.867\end{array}$

As we expect the dynamic and alpha static results are in perfect agreement. Statistically the $\mathrm{K}$ static method is in excellent agreement; K-eff agrees into the fourth digit and the removal lifetime to within $1 \%$. Note that due to the relationship,

$\alpha=\left[(K-1) / T_{R}\right]$ 
for $K$ close to unity, we expect a large uncertainty in small values $\alpha$, so that the differences we see here are acceptable for our use.

Problem \#2 - a homogeneous, fast neutron system, very super-critical: The same as Problem \#1, except that we will double the density of the material,

Spherical radius: $8.7407 \mathrm{~cm}$

Density: $\quad 37.4796$ grams/ce - the only difference from problem \#1

Composition: $\quad 922350.937695$ atoms

$92238 \quad 0.052053$ atoms

922340.010252 atoms

normalized to 37.4796 grams/cc overall density

Compared to problem \#1, here we move far away from critical, to a very super-critical, fast neutron system.

$\begin{array}{llll} & \text { Dynamic } & \text { K Static } & \text { Alpha Static } \\ \text { K-eff } & 1.660 & 1.582 & 1.660 \\ \text { Alpha/usec } & 144.7 & 111.9 & 144.7 \\ \text { Removal Time usec } & 4.559 \mathrm{e}-3 & 5.207 \mathrm{e}-3 & 4.559 \mathrm{e}-3 \\ \text { Median Neutron Energy MeV } & 1.060 & 0.711 & 1.060\end{array}$

The first figure below compares the flux spectrum calculated by dynamic and $\mathbf{K}$ static methods. As we pointed out above for a super-critical system such as this, compared to the correct dynamic method, the $\mathrm{K}$ static method biases the spectrum toward lower energies, by only combing neutron production. We can clearly see this effect in the below figure. The above table also shows this effect in that the median neutron energy calculated by the dynamic method is $1.060 \mathrm{MeV}$, whereas for the $\mathrm{K}$ static method it is only $0.711 \mathrm{MeV}$. This shift accounts for the significant difference in the time constants: dynamic 144.6/usec, K static 111.9/usec.

As we expect the dynamic and alpha static methods agree; both the plot below of the neutron production and the above tabulated results are statistically in perfect agreement. 


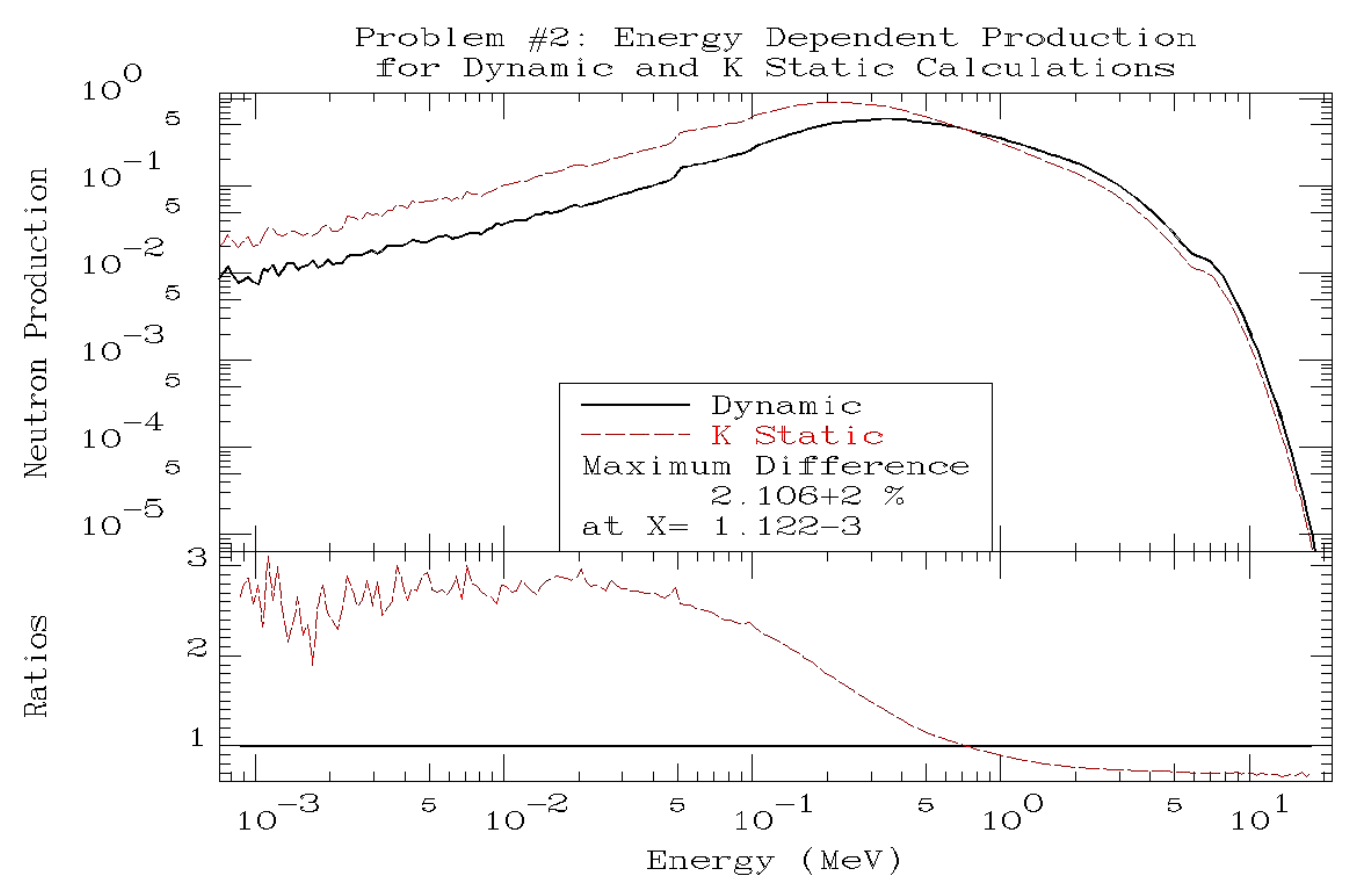

Problem \#2: dynamic vs. K Static production spectra

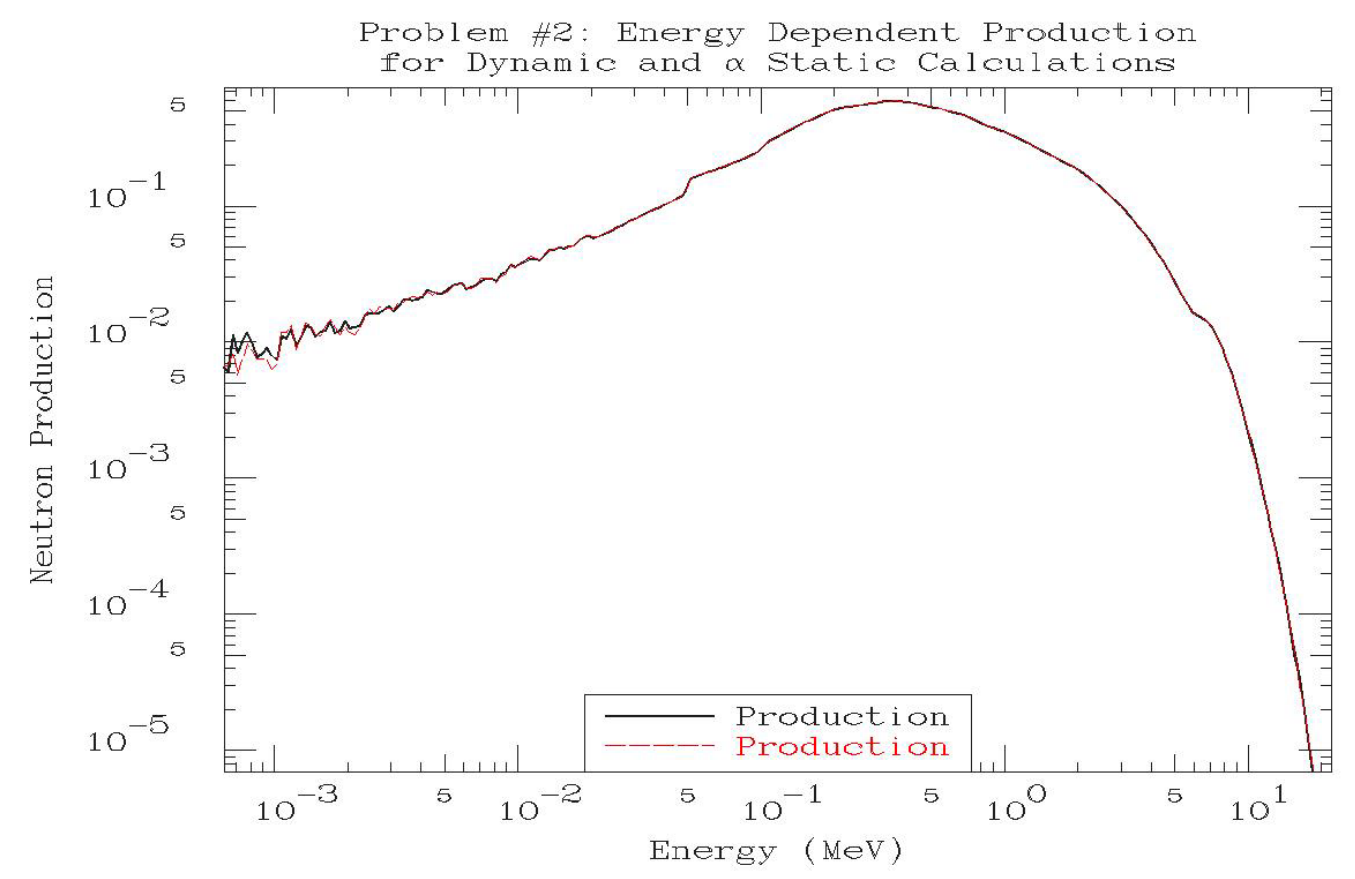

Problem \#2: dynamic vs. alpha Static production spectra 
Problem \#3 - a heterogeneous, fast neutron system, very super-critical: The same as Problem $\# 2$, except that we add a $30 \mathrm{~cm}$ radius sphere of water around the sphere of uranium,

Spherical radius: $8.7407 \mathrm{~cm}$

Density: $\quad 37.4796$ grams/cc

Composition: $\quad 922350.937695$ atoms

$92238 \quad 0.052053$ atoms

922340.010252 atoms

normalized to 37.4796 grams/cc overall density

Spherical radius: 8.7407 to $38.7407 \mathrm{~cm}$ cc - the only difference from problem \#2

Density: $\quad 1.0$ grams/ce

Composition: $\quad 10012.0$ atoms

80161.0 atoms

normalized to 1.0 grams/ce overall density

Compared to problems \#1 and \#2, which were homogeneous systems where everything is happening very fast, in this case we move to a heterogeneous system, where the time scale of the inner uranium sphere is much shorter than the time scale of the outer sphere of water.

$\begin{array}{llll} & \text { Dynamic } & \text { K Static } & \text { Alpha Static } \\ \text { K-eff } & 2.349 & 1.661 & 2.349 \\ \text { Alpha/usec } & 146.6 & 1.354 \mathrm{e}-2 & 146.6 \\ \text { Removal Time usec } & 9.203 \mathrm{e}-3 & 48.86 & 9.203 \mathrm{e}-3 \\ \text { Median Neutron Energy MeV } & 1.054 & 0.589 & 1.054\end{array}$

Based on the first below plot comparing neutron production calculated by the dynamic and K static methods, and the above tabulated results, we would never have guessed that these two sets of results are for exactly the same system using two different solution methods. The above results are not typos, e.g., the removal times really are: dynamic 0.0092 usec, K static 48.86 usec, not quite five orders of magnitude $\left(10^{5}\right)$ different.

In the first below plot we can see the high energy bias introduced by the K static method, but what is really striking is the bias in space. The long low energy tail of the $\mathrm{K}$ static results means that high energy neutrons escape from the uranium, slow down in the water, and then return back into the uranium causing low energy fissions. In contrast the dynamic solution shows virtually no low energy return from the water. The difference is due to "time". The dynamic results indicate that it takes far too much time for a neutron to leak from the uranium, slow down in the water, and return to the uranium to make any significant contribution to the flux in the uranium.

During this time the neutron flux has been exponentially increasing in the uranium, so that by the time any thermal neutrons return from the water their contribution is tiny compared to the "current" neutron flux in the uranium. Let us give a simple numerical 
example: a thermal neutron is usually described as having a speed of 2200 meters $/ \mathrm{sec}$, which means $0.22 \mathrm{~cm} /$ usec. Consider a neutron that has traveled out into the water and thermalized $30 \mathrm{~cm}$ into the water. We will ignore the time for the neutron to get to this location and thermalize in the water and only consider the fastest possible crow fly time in which it could travel back $30 \mathrm{~cm}$ to the inner uranium sphere, which is almost 150 usec. During this time the flux in the uranium will increase by $\operatorname{Exp}\left(\alpha^{*} t\right)=$ $\operatorname{Exp}(146 * 150)=\operatorname{Exp}(21,900)=A$ VERY BIG NUMBER!!!! If you think $30 \mathrm{~cm}$ is unreasonable try $3 \mathrm{~cm}$ : $\operatorname{Exp}(2,190)$, or even $0.3 \mathrm{~cm}$ : $\operatorname{Exp}(219)$. Get the idea? Neutrons simply cannot be thermalized and return to the central sphere of uranium in time to make any reasonable contribution to the flux.

The $\mathrm{K}$ static results do not consider time, and in addition in this case the $\mathrm{K}$ static method highly biases the neutron flux away from the uranium and into the water. This is an excellent example of where the $\mathbf{K}$ static method completely fails to solve the problem we posed.

Remember what we said above about the importance of other parameters. In this case if we had run a $\mathbf{K}$ static calculation to define $\mathrm{K}$ and removal time, to subsequently use as input to a dynamic calculation, our "guess" of the appropriate removal time for the dynamic calculation would have been off by almost a factor of $10^{5}$, which could prevent a dynamic calculation from running at all. In addition the $\mathrm{K}$ of 1.66 versus 2.34 could underestimate the rate of energy deposition due to fission.

Again as we expect, in this case the alpha static method is in perfect agreement with the dynamic method; both the below plot of the neutron production and the above tabulated results statistically are in perfect agreement with the dynamic results. Compared to the enormous thermal production predicted by the $\mathrm{K}$ static method, the alpha static method combs out the low energy spectrum, and produces complete agreement with the dynamic production spectrum. This is an excellent example of time absorption at work (see the appendix for details). Unlike the $\mathrm{K}$ static method, which ignores time, the alpha static has included the effect of the time it would take neutrons to return from the water, thereby suppressing the low energy spectrum. 


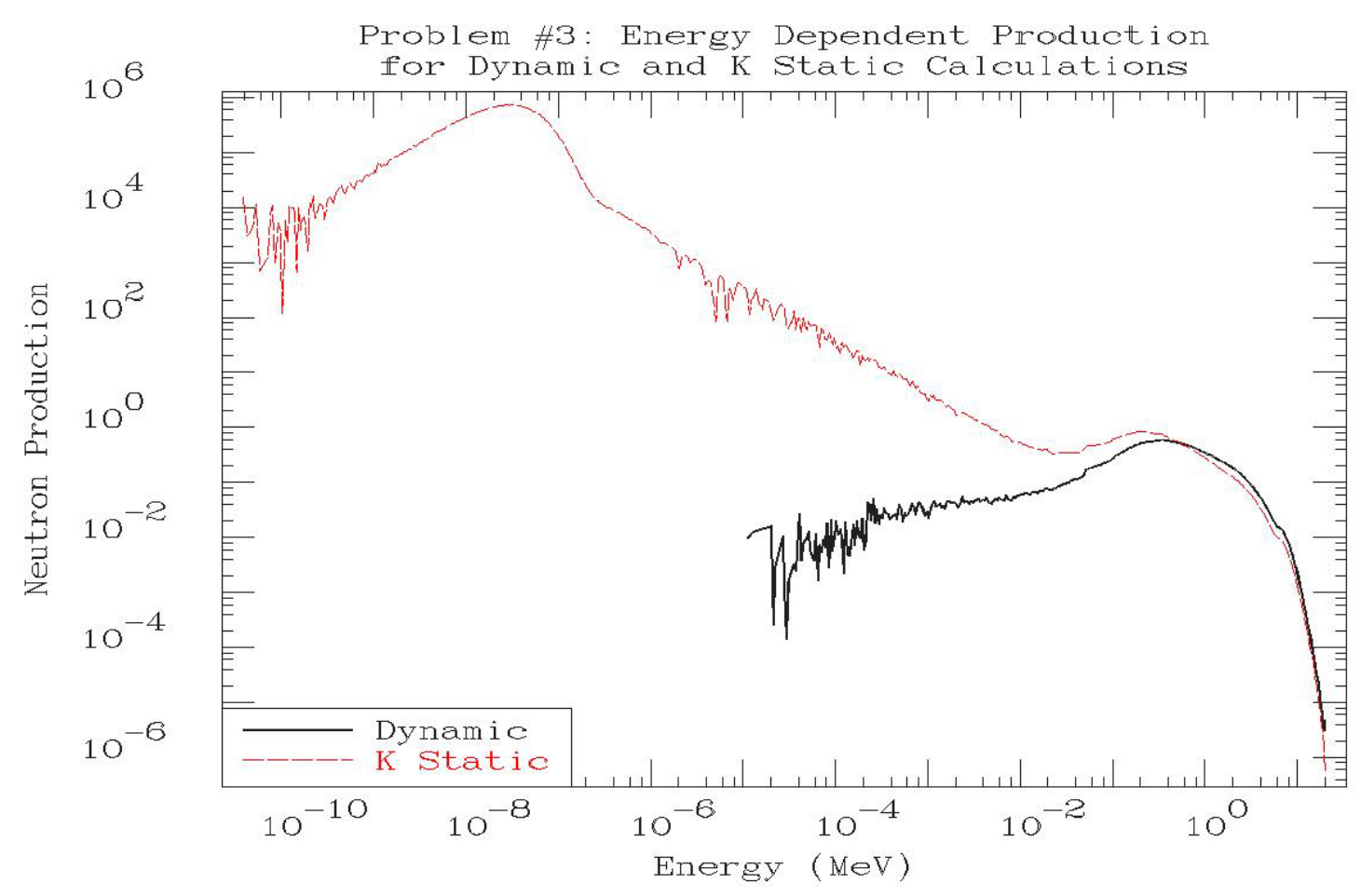

Problem \#3: dynamic vs. K Static production spectra

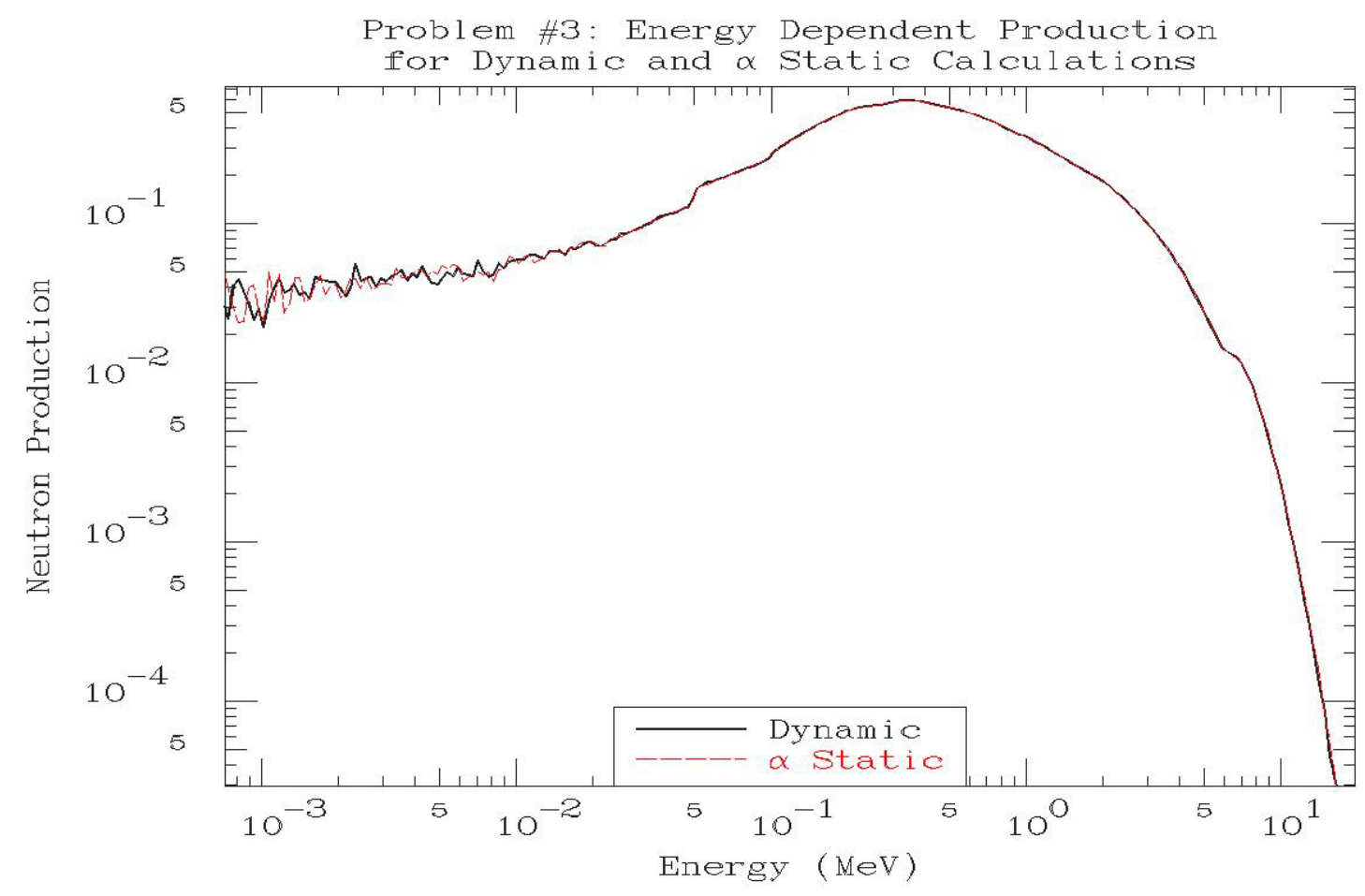

Problem \#3: dynamic vs. alpha Static production spectra 
Problem \#3b - same as Problem \#3, with reduced density to make the system critical This solution is included merely to show that the problem that $\mathrm{K}$ static method is solving is for a critical system that has little to do with the problem \#3 that we are interested in.

Spherical radius: $8.7407 \mathrm{~cm}$

Density: $\quad 13.1$ grams/cc - the only difference from problem \#3

Composition: $\quad 922350.937695$ atoms

$92238 \quad 0.052053$ atoms

922340.010252 atoms

normalized to 13.1 grams/cc overall density

Spherical radius: 8.7407 to $38.7407 \mathrm{~cm} \mathrm{cc}$ - the only difference from problem \#2

Density: $\quad 1.0$ grams/cc

Composition: $\quad 10012.0$ atoms

80161.0 atoms

normalized to 1.0 grams/cc overall density

$\begin{array}{llll} & \text { Dynamic } & \text { K Static } & \text { Alpha Static } \\ \text { K-eff } & 1.00032 & 1.00027 & 1.00032 \\ \text { Alpha/usec } & 3.08 \mathrm{e}-6 & 2.60 \mathrm{e}-6 & 3.08 \mathrm{e}-6 \\ \text { Removal Time usec } & 104.44 & 104.46 & 104.44 \\ \text { Median Neutron Energy MeV } & 0.348 & 0.348 & 0.348\end{array}$

Here we can see that this critical system has the same well thermalized spectrum as predicted by the $\mathrm{K}$ static method for problem \#3. This is because in this case the system being critical means virtually no time dependence. So that even though it may take a long time to happen, neutrons are continuously leaking from the uranium, slowing down in the water, and returning to the uranium, to cause the thermalized neutron production shown below.

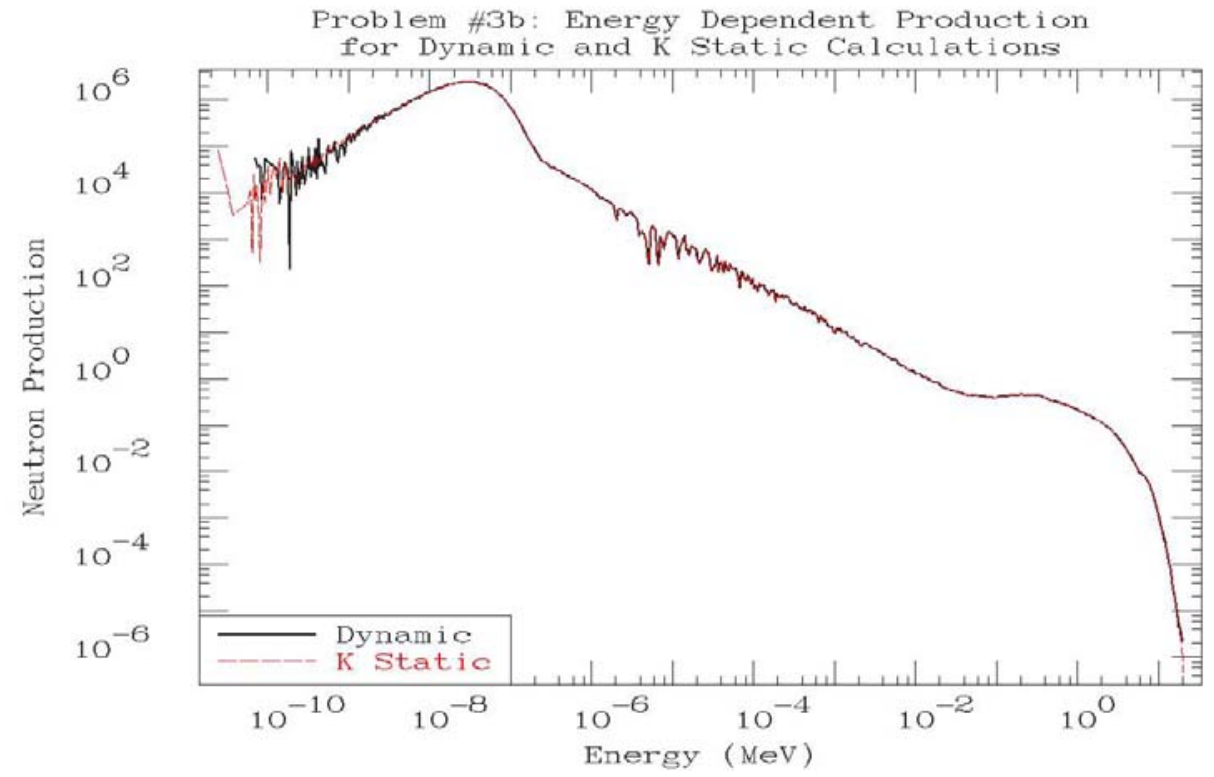

Problem \#3b: dynamic vs. K Static production spectra 
Problem \#4 - a homogeneous, thermal neutron system, very super-critical: The same as problem $\# 1$, except that we change the composition by replacing some of the uranium by water, i.e., 100 parts water to 1 part uranium,

Spherical radius: $8.7407 \mathrm{~cm}$

Density: $\quad 18.7398$ grams/cc

Composition: 922350.937695 atoms

922380.052053 atoms

922340.010252 atoms

1001200.0 atoms This is the only difference

8016100.0 atoms from problem \#1

normalized to the above overall density

Compared to the preceding problems, this problem has so much moderator mixed directly with the uranium that the neutron spectrum will be thermalized. What may come as a shock to some readers is that in this case we started with problem \#1 that was just about critical, replaced some of the uranium with water, and end up with a highly super-critical system!!! Surprise, surprise - all this tells us is that a solid sphere of uranium is not the optimum spatial configuration for criticality, which we hope doesn't come as a surprise to anyone, since this has been known since the 1940's.

$\begin{array}{llll} & \text { Dynamic } & \text { K Static } & \text { Alpha Static } \\ \text { K-eff } & 1.724 & 1.771 & 1.724 \\ \text { Alpha/usec } & 0.653 & 0.675 & 0.653 \\ \text { Removal Time usec } & 1.108 & 1.141 & 1.108 \\ \text { Median Neutron Energy MeV } & 4.680 \mathrm{e}-8 & 3.715 \mathrm{e}-8 & 4.680 \mathrm{e}-8\end{array}$

From the above table the first thing to note about this system is that the neutron removal time is over a hundred (100) times longer than in the first three problems, i.e., everything is happening much slower in this system. In this thermal system the fast neutrons produced by fission take a relatively long time to slow down and eventually contribute to the thermal spectrum we see in the below plots.

From the above table we can see that the K Static results agree very well with the dynamic results for this thermal system. Remember that $\mathrm{K}$ Static biases the flux toward lower energies by combing fast neutron production. But in this case from the below figure we can see that the fast neutron flux is quite small, and the system is really driven by thermal neutrons. The net effect is that although we can see the K static bias of the high energy flux (it is definitely less than the dynamic result) this has little effect on the integral parameters of the system. This illustrates why the $\mathrm{K}$ static method works well for thermal, homogeneous systems.

As we expect, the alpha static method results agree with the dynamic method results; both the below plot of the neutron production and the above tabulated results are in perfect agreement with the dynamic results. 


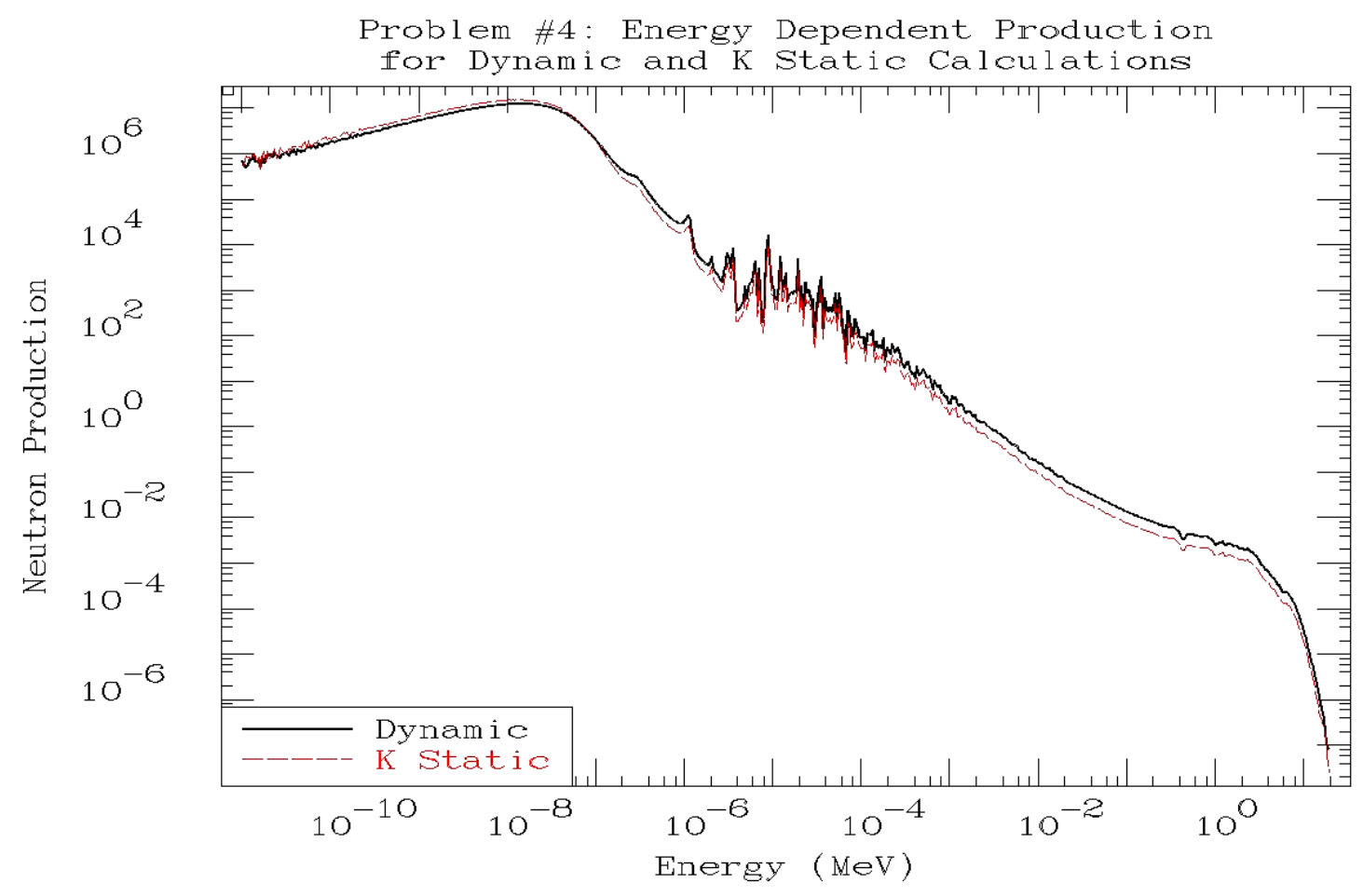

Problem \#4: dynamic vs. K Static production spectra

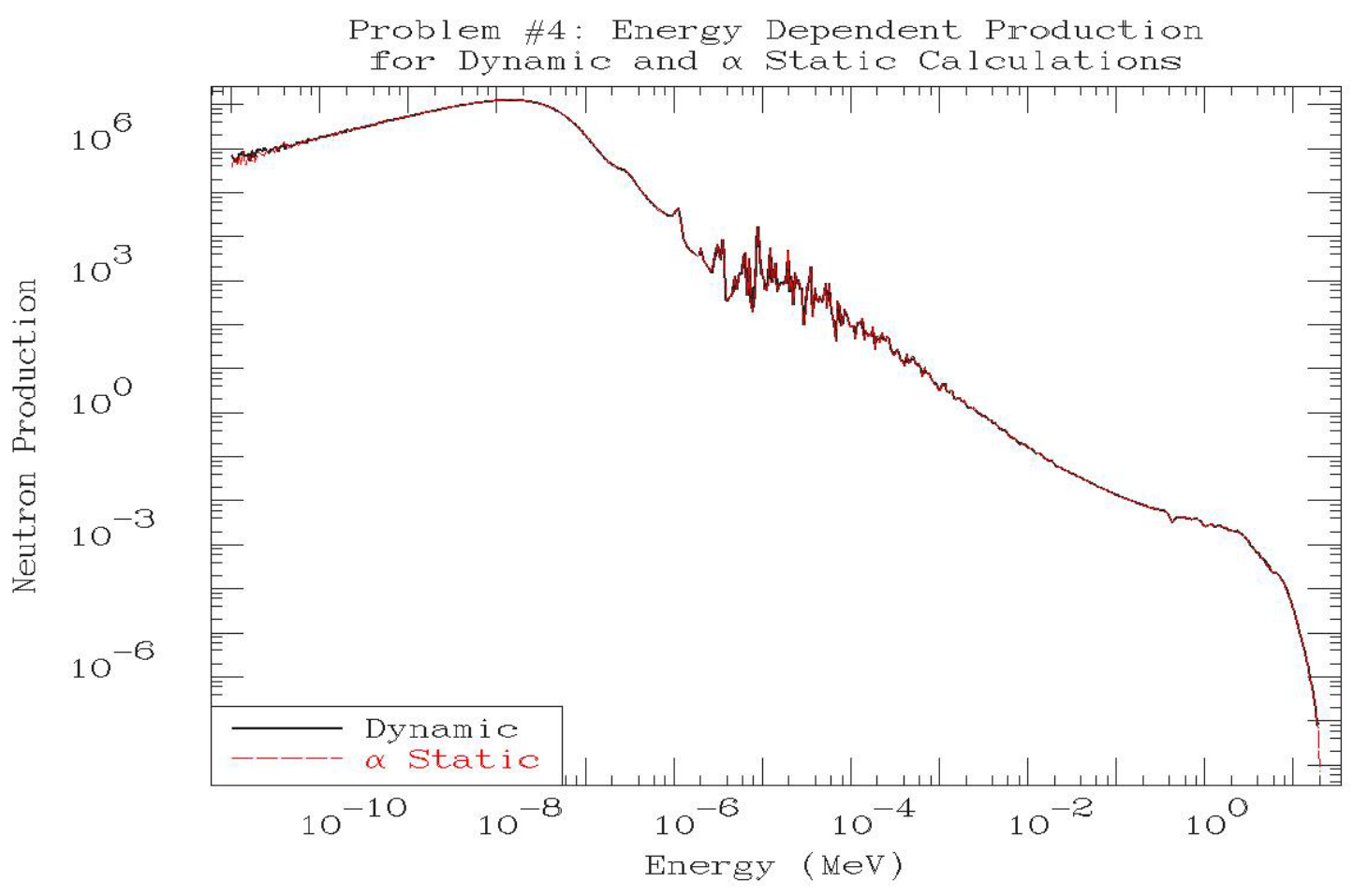

Problem \#4: dynamic vs. alpha Static production spectra 
Problem \#5 - a homogeneous, fast neutron system, very sub-critical: The same as Problem \#1, except that we will half the density of the material,

Spherical radius: $8.7407 \mathrm{~cm}$

Density: $\quad 9.3999$ grams/cc - the only difference from problem \#1

Composition: $\quad 922350.937695$ atoms

$92238 \quad 0.052053$ atoms

922340.010252 atoms

normalized to 9.3999 grams/cc overall density

Compared to problem \#1, here we move far away from critical, to a very sub-critical, fast neutron system.

$\begin{array}{llll} & \text { Dynamic } & \text { K Static } & \text { Alpha Static } \\ \text { K-eff } & 0.763 & 0.528 & 0.763 \\ \text { Alpha/usec } & -1.048 & -79.53 & -1.048 \\ \text { Removal Time usec } & 0.226 & 5.93 \mathrm{e}-3 & 0.226 \\ \text { Median Neutron Energy MeV } & 8.46 \mathrm{e}-2 & 1.086 & 8.46 \mathrm{e}-2\end{array}$

The first figure below compares the neutron production calculated by dynamic and $\mathrm{K}$ static methods. As we pointed out above, in this case because the system is sub-critical, compared to the correct dynamic method, the K static method biases the spectrum toward higher energies; in this case combing produces more high energy neutrons, thereby biasing the spectrum toward higher energies. We can clearly see this effect in the below figure. The above table also shows this effect in that the median neutron energy calculated by the dynamic method is $0.0846 \mathrm{MeV}$, whereas for the $\mathrm{K}$ static method it is $1.086 \mathrm{MeV}$. This shift accounts for the significant difference in the time constants: dynamic -1.048/usec, K static -79.53/usec.

Remember that these neutron production curves are normalized per neutron produced, when integrated over the entire neutron production energy range. The fact that the below $\mathrm{K}$ settle production is roughly twice as high as the dynamic production, indicates that although the energy range shown includes all of the $\mathrm{K}$ settle production, it only includes about half of the dynamic production. The remaining dynamic production is at lower energies. This lower energy production does not appear in the $\mathrm{K}$ settle production because of its bias toward producing higher energy neutrons.

As we expect the dynamic and alpha static methods agree; both the plot below of the neutron production and the above tabulated results are statistically in perfect agreement. 


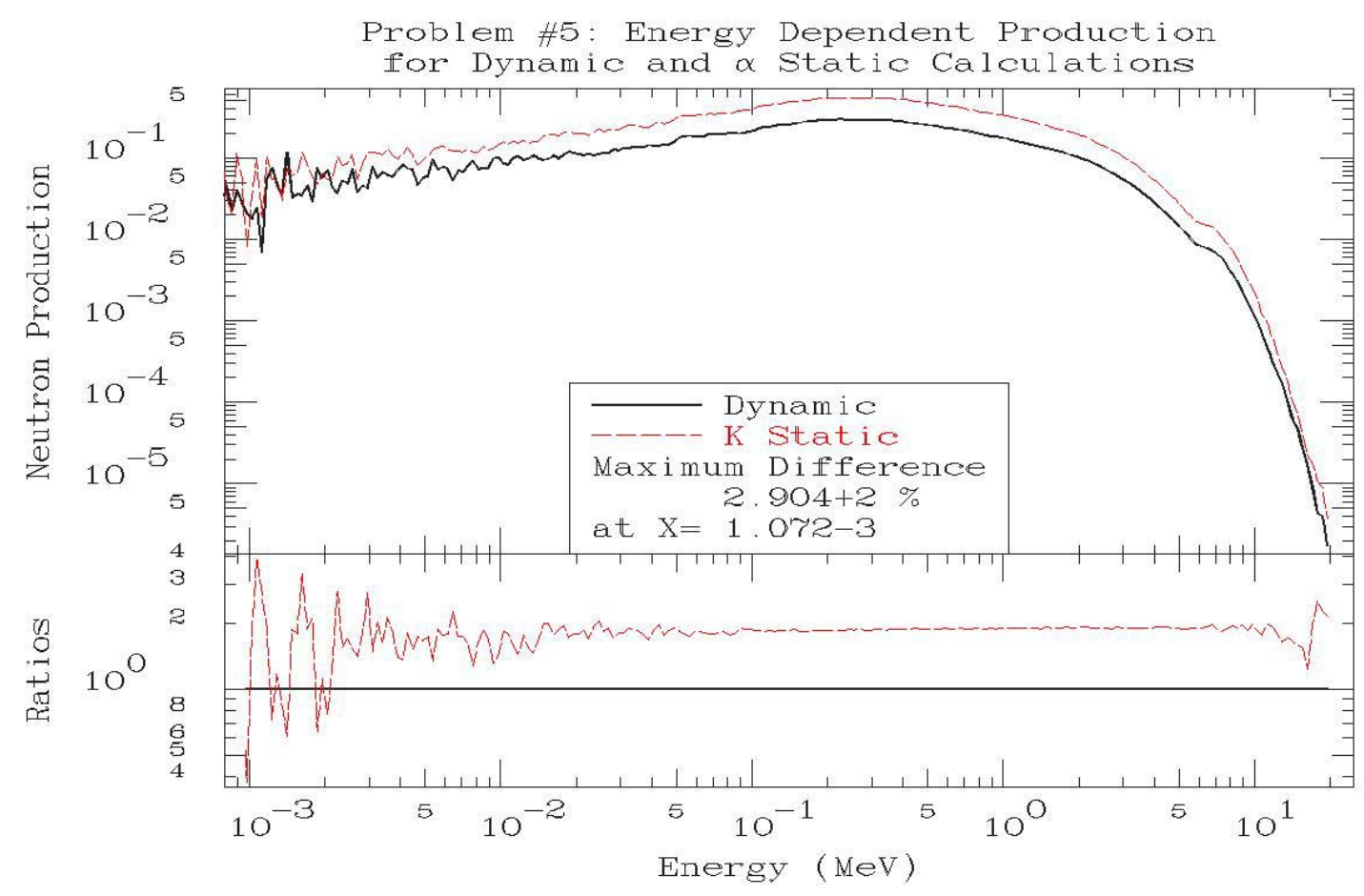

Problem \#5: dynamic vs. K Static production spectra

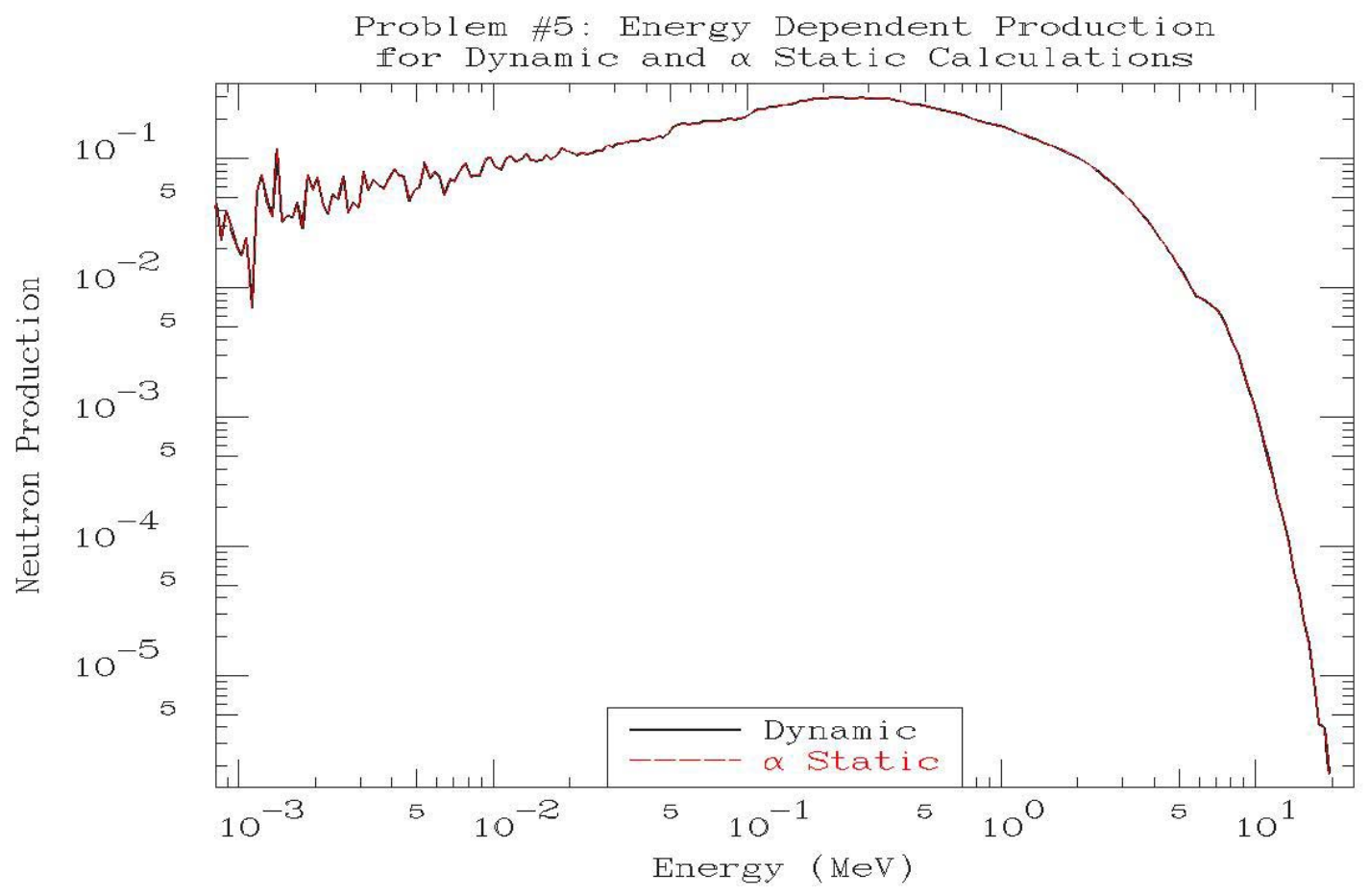

Problem \#5: dynamic vs. alpha Static production spectra 


\section{Back to Reality}

The above results only apply to the problem as we originally described it. In reality there are at least two items that complicate real problems. Note, that these items complicate real problems, but they do not change the above conclusions, which are based strictly on how each methods combs neutrons; since this is so important we will repeat this statement below. The two complicating items are,

First are delayed neutrons. We are not going to go into detail here; see the appendix for a description of how delayed neutrons affect problems. We will merely mention here that generally the idea of a simple, single exponential based on the behavior of prompt neutrons, only applies to systems that are highly super-critical. That's why the problems that we used above were all of this type, where we have a rather large, positive time constant, $\alpha$. Therefore users should beware of giving any physical significance to time constants calculated for any systems without including delayed neutrons.

Next is time independent media. Experience tells us that in a nuclear reactor even small variations of reactivity from $\mathrm{K}$-eff $=1$, cause the media to respond. For example, when K-eff increases the temperature of the medium increases, and typically the cross sections Doppler broaden and the media thermally expands. This response should also affect the above idealized results based on fixed, time independent media. Let's be realistic: above we examined problems with very high K-eff, but we did not address the question ofhow one would start from a sub-critical system and manage to change the system to make it so highly super-critical, without the medium being time dependent. That's just not realistic; time dependent media have to be considered.

In order to make our point concerning the differences between dynamic and static criticality problems we tried to keep the above presentation as simple as possible by only considering idealized problems that do not include delayed neutrons and time dependent media. The important point for readers to realize is that whether or not delayed neutrons and time dependent media are included in a calculation does not change the above conclusions, which are based strictly on how each method combs neutrons, leading to the conclusions that,

1) dynamic and alpha static methods produce the same correct answers,

2) dynamic and $\mathrm{K}$ static methods do not produce the same answer.

\section{Where can the K Static Method be Used?}

Since this is such an important point, we repeat here what we said near the beginning of this report.

The $\mathrm{K}$ static method described in this paper has been successfully used for criticality calculations for over 50 years. It has been the workhorse of the criticality business, and has been widely documented in virtually every textbook on the subject, and we are sure that it will continue to be just a widely used in the future. Here we point out that when 
properly used this method is accurate, practical and very economical for use in criticality calculation.

But we also document where this method can be "properly" used, i.e., if you misuse any method the answers you get are questionable. In the case of the $\mathrm{K}$ static method it is very accurate for systems close to critical, i.e., K-eff $\sim 1$. It is also very effective if you wish merely to qualitatively define whether a system is sub or super-critical, as well as for monitoring the approach of systems to or from an exactly critical state. However, if you are interested in systems that are far from critical, the $\mathrm{K}$ static method may not be accurate, and the difference between the true and accuracy solution and the $\mathrm{K}$ static method solution grows as a system becomes progressively further from critical.

\section{Conclusion}

We can only hope that the descriptions and the few simple examples presented here prove that dynamic and alpha static methods are equivalent, and if properly used give the same correct answers. In contrast, dynamic and K static criticality calculations do not give the same results, and the results presented here illustrate the magnitude of the differences in results, which can be very large, as in example \#3. We also hope that the discussion of all methods as time dependent problems help to explain that mathematically the only different between the three methods discussed here is how each combs neutrons to maintain population control,

The dynamic method combs the neutron population only at the end of each time step, uniformly in space, energy and time by a factor of, $\operatorname{Exp}\left(\alpha^{*} t\right)$, to change the neutron population back to what it was at the beginning of a time step, resulting in an unbiased sample that can be used to continue the calculation into successful time intervals. Again we will mention that comparisons to true analog calculations without combing demonstrate that the dynamic method with combing gives exactly the same answer.

The K static method combs neutron production throughout each time step, to obtain the same number of neutrons at the end of the time step as existed at the beginning of the time step. Compared to the correct dynamic method, this biases the neutron spectrum in both energy and space, e.g., for a super-critical system it softens the spectrum and shifting the flux away from fissile materials.

The alpha static method combs neutron absorption throughout each time step, uniformly in space, energy and time by a factor of, $\operatorname{Exp}\left(\alpha^{*} t\right)$, to obtain the same number of neutrons at the end of the time step as existed at the beginning of the time step. This is exactly equivalent to what the dynamic method does, which is why the dynamic and alpha static results agree.

Note, that for a system that is exactly critical no combing is required, and all three methods are equivalent. As a system moves progressively further away from its critical 
state the amount of combing required by each of these methods increases, causes the $\mathrm{K}$ static method solution to differ progressively more from the true solution..

Since this is the entire point of this paper let us repeat the conclusions,

1) dynamic and alpha static criticality calculations are identical and correct, so that if properly used TART using the dynamic method and MCNP using the alpha static method should produce the same answers.

2) The textbook K static method that we were all taught in school, is generally only accurate for systems that are close to critical; as we have seen above, in other cases this method can be very inaccurate.

The bottom line is that for systems close to critical all of these methods give the essentially the same answer, and because of its speed and strong convergent properties the $\mathrm{K}$ static method is still the most efficient for such problems. It is also the most efficient method if you only want to qualitatively determine whether a system is sub or super-critical, without too much concern about a precise value for $\mathrm{K}$.

It is only when a system is far from critical that the $\mathrm{K}$ static system fails. In this case for homogeneous systems, if the system is slow the $\mathrm{K}$ static method can still be accurate. For heterogeneous systems K static methods can have accuracy problems, e.g., see even the very simple problem \#3.

Historically the dynamic method has been avoided in preference to the $\mathrm{K}$ static method because it was thought that the dynamic method is too expensive, in terms of computer running time. It is still true that the $\mathrm{K}$ static calculation is still much faster to run than a dynamic calculation, but due to the fairly recent increase in available inexpensive computer power, the dynamic method is now practical for use in many applications that we couldn't afford to attempt just a few short years ago, e.g., see the improved speed of TART on a variety of computers, over the last few years see,

http://www.llnl.gov/cullen1/speed.htm

As you can see from this table, in the last few years we have seen an enormous increase in the availability of inexpensive computing power. We would hope that at least some of this increase is devoted to improving the accuracy of results, as opposed to merely running the same old methods faster. In deciding which methods you use in your applications we highly recommend that you remember Howerton's first law: "We are in no rush for the wrong answer" [5].

\section{References}

[1] "TART 2002: A Coupled Neutron-Photon, 3-D, Combinatorial Geometry, Time Dependent Monte Carlo Transport Code", UCRL-ID-126455, Rev. 4, (June 2003), Lawrence Livermore National Laboratory, by Dermott E. Cullen 
[2] "MCNP5 - A General Monte Carlo N-Particle Transport Code, Version 5," LA-UR03-1987 (April 24, 2003), Los Alamos National Laboratory, by the X-5 Monte Carlo Team.

[3] "MERCURY User's Guide", Version b1, (in press) Lawrence Livermore National Laboratory, by Richard J. Procassini and Janine M Taylor.

[4] “AMTRAN User's Guide”, (in press) Lawrence Livermore National Laboratory, by Christopher J. Clouse.

[5] Robert J. Howerton, private communication (1975)

\section{Acknowledgements}

We thank the following people who read earlier versions of this paper, and who supplied extremely useful constructive criticism, that led to important improvements in the final paper; they are in alphabetical order: Forrest Brown (LANL), Maurice Greene (ORNL), Dave Heinrichs (LLNL), Ernest Plechaty (LLNL), and Ray Tolar (LLNL),

\section{Appendix}

\section{Delayed Neutrons}

All of the above discussions, equations, calculations, and conclusions are based on ignoring delayed neutrons. When delayed neutrons are included the picture changes dramatically. As far as this paper is concerned the important point to realize is that delayed neutrons dramatically change the time dependent behavior of a system. Only in highly super-critical systems do we expect to see the simple, single exponential variation of the flux based solely on the behavior of prompt fission neutrons, described above. In other cases delayed neutrons can be the dominant factor in defining the time dependence of a system. When delayed neutrons are included the time scale of neutron transport problems can change from the fast neutron removal time characterized by microseconds to the millisecond to second time scale on which delayed neurons are emitted after fission. So if your calculations do not include the effects of delayed neutrons, use caution in interpreting results.

Here we will merely outline the changes in the problem when delayed neutrons are included. For starters, equation [1], is instantaneous, in the sense that the equation only involves the current time $(t)$, e.g., we assume all neutrons emitted in fission are emitted at the instant that the fission occurred,

$\frac{1}{v} \frac{\partial N}{\partial t}+\Omega^{*} \nabla N+\Sigma t^{*} N=\iint[<v>* \Sigma f+\Sigma s] F\left(E^{\prime}, \Omega^{\prime}->E, \Omega\right) N d E^{\prime} d \Omega^{\prime}+S$

With delayed neutrons this changes to include an integral over all earlier times $\left(t^{\prime}\right)$, 


$$
\frac{1}{v} \frac{\partial N}{\partial t}+\Omega^{*} \nabla N+\Sigma t^{*} N=\iiint[<v>* \Sigma f+\Sigma s] F\left(E^{\prime}, \Omega^{\prime}, t^{\prime}->E, \Omega, t\right) N d E^{\prime} d \Omega^{\prime} d t^{\prime}
$$

where the flux at the current time $(t)$ is now connected to the flux at all earlier times $\left(t^{\prime}\right)$. This is because fission at some earlier time $\left(t^{\prime}\right)$ can now result in the emission of a delayed neutron at the current time $(t)$. This equation can be simplified by connecting the flux at the current time $(t)$ not to the multi-dimensional flux at earlier times $\left(t^{\prime}\right)$, but rather to the time dependence of the spatial density of fission product precursors that emit delayed neutrons. We assume that the delayed neutrons are emitted by a defined set of $L$ fission products.

The equation defining the density of each fission product $k$ is,

$$
\frac{\partial C k}{\partial t}(r, t)=-\lambda k^{*} C k(r, t)+\int<v d k>\Sigma f\left(r, E^{\prime}\right) N 0\left(r, E^{\prime}, t\right) d E^{\prime}
$$

Here the first term defines the rate of decay of the fission product and the integral over energy defines the production of fission product $k$ due to fission in a system; a complete description includes a third term to account for burn-up of fission products.

The neutron source produced by these fission products is,

$S d(r, E, t)=\sum_{k=1}^{L} \lambda k^{*} C k(r, t) \chi d k(E)$

Where the decay of fission product $k$ emits neutrons with an energy spectrum $\chi d k(E)$.

The total number of delayed neutrons emitted per fission is the sum over all fission products emitting delayed neutrons,

$<v d(E)>=\sum_{k=1}^{L}<v d k(E)>$

$C k(r, t) \quad$ - density of fission product $k$

$\lambda k \quad-$ decay constant for fission product $k$

$<v d k>\quad$ - production of fission product $k$ per fission

$\chi d k(E) \quad$ - spectra of neutrons emitted by decay of fission product $k$

The coupled set of equations to solve are then the above equations for the density of fission product, and our equation [1] now in its original instantaneous form, but including the emission of the delayed neutrons due to the decay of fission products $S d(r, E, t)$, 


$$
\frac{1}{v} \frac{\partial N}{\partial t}+\Omega * \nabla N+\Sigma t^{*} N=\iint[<v p>* \Sigma f+\Sigma s] F\left(E^{\prime}, \Omega^{\prime}->E, \Omega\right) N d E^{\prime} d \Omega^{\prime}+S d
$$

In this form we can see that even in the absence of any other neutron sources, when equation [1] predicts that the flux would rapidly drop to essentially zero, the presence of the delayed neutron source $S d(r, E, t)$ will make the neutron flux persist for relatively long periods of time, i.e., milliseconds to seconds and beyond.

I'll merely mention that this picture is further complicated by the delayed emission of neutrons by processes other than fission, e.g., photonuclear reactions in $\mathrm{Be} 9, \mathrm{D} 2, \mathrm{Li} 6$, C13; see the below books for details.

As far as this paper is concerned the important point to note is that for any system involving fission unless you are dealing with a highly super-critical system the idea that the flux in a system can be characterized by one simple, single exponential based strictly on the behavior of prompt fission neutrons is questionable; so use care in drawing any conclusions based on this assumption.

\section{However, this effect does not effect the conclusion that for time dependent systems dynamic and alpha static methods are correct, and $\mathrm{K}$ static is not, particularly for systems that are far from critical.}

More details on delayed neutrons are beyond the scope of this paper. For further details concerning delayed neutrons see the now classical book on the subject,

"Physics of Nuclear Kinetics" by G. Robert Keepin, Addison-Wesley Publishing Company, Inc (1965)

and the more recent book,

"Introduction to Nuclear Reaction Kinetics", by Daniel Rozon, Polytechnic International Press (1998).

\section{Hyperbolic Equation}

Equation [1] is a hyperbolic equation with real characteristics, where the three terms on the left hand side of the equation are exactly correlated to define the real characteristics. To see this we can re-write the equation with the three terms on the left written as a single term,

$$
\frac{d}{d \xi}\left\{N(R-\Omega \xi, \Omega, E, t-\xi / v) * \operatorname{Exp}\left[\Sigma t^{*} \xi\right]\right\}=\operatorname{Exp}\left[\Sigma t^{*} \xi\right] \iint[<v>\Sigma f+\Sigma s] F^{*} N d E^{\prime} d \Omega^{\prime}
$$


where $\xi$ is the distance traveled by a neutron along its path. In this form we can see that the motion in space $[\Omega * \nabla N]$ is exactly correlated to the motion in time $\left[\frac{1}{v} \frac{\partial N}{\partial t}\right]$. This is the form in which a truly time dependent Monte Carlo code solves this equation, e.g., we select a distance of travel (D) to the next event, we advance our particle in space a distance $\mathrm{D}$, and we advance the particle in time $\mathrm{D} / \mathrm{v}$, to maintain the exact correlation.

The assumption that the flux throughout the entire system can be characterized by a simple, single exponential behavior,

$$
\frac{\partial N}{\partial t}(r, \Omega, E, t)=\alpha^{*} N(r, \Omega, E)
$$

breaks this exact correlation and changes the nature of the equation. Instead of the original hyperbolic equation, with real characteristics defining the lines along which neutrons can move in space and time in a correlated manner, we now have a parabolic equations in which there is no net correlated propagation in (space, time) from one point in the system to another. In principle, when we solve a static problem time does not appear in the equation, and this assumption locks the system into a form with instantaneous propagation, so that the flux instantaneously rises and falls at exactly the same rate throughout the entire system, in space, energy, and direction.

Making this assumption of exponential variation at the differential level is quite different from our observing that for the integral system as a whole, we expect it to eventually evolve into a fundamental mode with a single, simple exponential variation. In making this observation for the overall system, when we perform a dynamic criticality calculation, we do not introduce any constraints that may bias the flux distribution and prevent it from evolving into its true fundamental mode distribution.

The point to realize that the true analog calculation can solve equation [1] to define the actual time dependent variation of the neutron flux without introducing any additional constraints, such as combing; the alpha static method cannot perform this general calculation. Only in the case of performing a fundamental mode criticality calculation are the dynamic and alpha static methods equivalent, since it is only in this case that we can assume simple exponential variation of the flux uniformly throughout the entire system.

The following section explains additional constraints placed on a system when we assume it is in its fundamental mode.

\section{Time Absorption or Production}

It is important to understand that the assumption that the system is in its fundamental mode introduces several important, related effects. First it means that there is a separation in (space, energy, direction) and (time); that's obvious from the definition. But there is an important related effect that many readers do not understand - the concept of time absorption. Separation of (space, energy, direction) and (time) does not mean that with 
time neutrons cannot move in space; this is secular equilibrium, where the rate of transfer to each space point from every other space point is constrained to maintain this secular equilibrium.

Consider a system that is really in its fundamental mode; then time is an important factor, both from the viewpoint of local and global growth or decay of the flux, and from the time it takes a neutron to transit from one point in a system to another. For a super-critical system the time absorption accounts for the fact that if a neutron leaves point $A$ and travel to point $\mathrm{B}$, when it starts its journey at point $\mathrm{A}$ the local flux has a given value, but while it is traveling the flux is exponentially increasing. The time absorption accounts for the change in the time dependent flux as neutrons transit from one point in the system to another. When the neutron arrives at point B its "value" should reflect the fact that when it left point $\mathrm{A}$ the flux was much lower than the current flux at point $\mathrm{B}$. This is exactly the effect introduced by time absorption, e.g., the probability of surviving the transit from point A starting at time $\mathrm{T} 1$ and arriving at point $\mathrm{B}$ at time $\mathrm{T} 2$, is reduced by the rate of growth of the flux during the time interval T1 to T2, $\operatorname{Exp}[\alpha(T 2-T 1)]$. Note that if the distance from point $\mathrm{A}$ to point $\mathrm{B}$ is $\mathrm{D}$, and the neutron is traveling with a speed $v$, the change in the flux $\operatorname{Exp}[\alpha(T 2-T 1)]$ is in exactly the form that appears in the alpha static method $\operatorname{Exp}\left[\alpha^{*} D / v\right]$, even though the alpha static equation does not explicitly include time.

Similarly for the sub-critical system time production accounts for the decrease in the flux with time as a neutron transit from point $A$ to point B. Constraining the flow of neutrons in this manner is the only way that we can allow neutron transport and at the same time maintain the secular equilibrium needed for separation of (space, energy, direction) and (time).

\section{Definition of Neutron Removal Rate and Time}

In order to properly define the system parameters we are interested in it is important to focus on the original system, without any combing. Obviously we are not interested in K $=1$ for the static system; we are interested in the K-eff for the real system. Our original equation integrated over space, energy, and direction, for the entire system is,

$$
\frac{d N_{0}}{d t}=v[<v>* \Sigma f-(L+\Sigma a)] N_{0}
$$

For this time dependent system we can define the system averaged absorption as,

$$
\Sigma a=\frac{\int \Sigma a(r, E) N(r, E, \Omega, t) d r d E d \Omega d t}{\int N(r, E, \Omega, t) d r d E d \Omega d t}
$$

Even when the geometry is fixed, in general this will be a time dependent quantity, due to the time dependence of the flux, as it evolves from the initial source distribution. 
However, once the flux is in its fundamental mode this equation is simplified, and the system averaged absorption becomes time independent,

$$
\Sigma a=\frac{\int \Sigma a(r, E) N(r, E, \Omega) \operatorname{Exp}\left[\alpha^{*} t\right] d r d E d \Omega d t}{\int N(r, E, \Omega) \operatorname{Exp}\left[\alpha^{*} t\right] d r d E d \Omega d t}=\frac{\int \Sigma a(r, E) N(r, E, \Omega) d r d E d \Omega}{\int N(r, E, \Omega) d r d E d \Omega}
$$

Note that since exactly the same time dependence appears in both numerator and denominator, it cancels out, leaving results that are independent of time. Similarly for the neutron production or leakage,

$$
<v>\Sigma f=\frac{\int<v(r, E)>\Sigma f(r, E) N(r, E, \Omega) d r d E d \Omega}{\int N(r, E, \Omega) d e d E d \Omega}
$$

Be aware that starting from an arbitrary source distribution the system will generally not initially be in its fundamental mode. Only after the passage of some time will it eventually relax into its fundamental mode. This means that the above integrals over time must be restricted only to the time when the system is in its fundamental mode. In a Monte Carlo calculation we accomplish this by the use of settle cycles, to allow time for the system to relax, or settle, into its fundamental mode. Only after these settle cycles do we begin to accumulate statistics to define the above integrals, i.e., the time integrals in the above equations begin after the settle cycles.

These are the quantities we are actually interested in, and in a Monte Carlo calculation they correspond to the actual absorption and production, again ignoring any effects introduced by combing. So for example, in the K static method in order to define the actual K-eff, we are interested in the actual production, $\langle v\rangle \Sigma f$, absorption, and leakage, not the modified production, $\langle v\rangle \Sigma f / K$, that we use only during combing for population control.

In a Monte Carlo calculation each neutron can be treated independently, in either a static or dynamic criticality calculation. The average removal time is then merely the sum of the time between "creation" of a neutron as a source or by fission, until it either leaks from the system or it is absorbed in the system (there are no other possibilities), divided by the number of neutrons that leak or are absorbed.

Let us say this again in a slightly different way: in defining any rate of events we measure all of the time involved and divide by the number of events that occur during this time. In the specific case of neutron transport, measure the total time that neutrons spend moving about. The rate of any type of event is then this single time divided by the number of events that occurred during this time; we can use this to define the rate of absorption, leakage, removal, production, etc. The average time for each event is then merely the reciprocal of the rate, e.g., the average removal time is the reciprocal of the average removal rate. 
It is important to note that this definition has nothing to do with whether the system is super or sub-critical, homogeneous or heterogeneous, fast or thermal neutrons. Whatever type of system is involved we need merely sample a neutron from the distribution of neutrons in space, energy, and direction, and keep track of the time until it is removed. The sum of all such times and the number of neutrons removed then defines the neutron removal time we are interested in.

Since this is so important, let us repeat that in defining the removal time do not become confused between real neutron removal and neutrons that are combed in order to maintain population control. For example in using the alpha static method for a highly supercritical system we will comb out and discard many neutrons using $\alpha / v$ "absorption" (actually Russian roulette), in order to maintain population control. In order to define the actual removal time of a neutron for the system it is important that you not include the combed neutrons in the total of neutrons removed. Similarly in using the K static method for a highly super-critical system we will comb out and discard many neutrons using $\langle v\rangle / K$, in order to maintain population control. In order to define the actual production it is important that you use the actual production $\langle v\rangle$, rather than the combed production $\langle v\rangle / K$.

\section{Definition of the Effective neutron Multiplication Factor, K-eff}

As described above, once a system is in its fundamental mode we can define time independent production and removal of neutrons, from which we can in turn define a time independent effective neutron multiplication factor, K-eff,

The effective neutron multiplication factor, K-eff, is defined as the number of neutrons produced per neutron removed,

$K=<v>* \Sigma f /(L+\Sigma a)$

In a Monte Carlo calculation each neutron can be treated independently, in either static or dynamic criticality calculations. The average $\mathrm{K}$ is then merely the sum of all neutrons produced ( $v$ neutrons per fission), divided by the number of neutrons that leak or are absorbed (removed $=$ absorbed plus leaked). Note that absorption included fission, which causes one neutron to be absorbed, and produces $\langle v\rangle$ neutrons.

It is important to note that this definition has nothing to do with whether the system is super or sub-critical, homogeneous or heterogeneous, fast or thermal neutrons. Whatever type of system is involved we need merely sample a neutron from the distribution of neutrons in space, energy, and direction, follow it until it is removed, and determine how many neutrons are produced when it is removed. The sum of all such neutron production and the number of neutrons removed then defines the reactivity $\mathrm{K}$ we are interested in.

In defining the reactivity do not become confused between real neutron removal and production and neutrons that are combed in order to maintain population control. For example in using either the $\mathrm{K}$ or alpha static method for a highly super-critical system we 
will comb out and discard many neutrons in order to maintain population control. In order to define the actual K-eff of the system it is important that you not include the combed neutrons in the total of neutrons removed or produced. In the simplest possible example to illustrate how silly it would be to include combed neutrons, in all static cases if you include the combed absorption and production, you will always find $\mathrm{K}$-eff $=1-$ because that is what $\mathrm{K}$ in the $\mathrm{K}$ static method, and alpha in the alpha static method are defined to achieve.

\section{Definition the time constant $\alpha$}

In a dynamic criticality calculation we can calculate $\alpha$ directly, based on our knowledge of the time dependent flux, $N_{0}(t)$,

$$
\begin{aligned}
& N_{0}(t 2)=N_{0}(t 1) * \operatorname{Exp}[\alpha(t 2-t 1)] \\
& \alpha=\log \left[N_{0}(t 2) / N_{0}(t 1)\right] /(t 2-t 1)
\end{aligned}
$$

This relationship is valid for any two different times.

In a dynamic or static calculation, by treating each neutron independently, we will obtain consistent definitions of both reactivity $(K)$ and neutron removal time $\left(T_{R}\right)$, which is all we need to uniquely define a consistent time constant $(\alpha)$,

$\alpha=\left[(K-1) / T_{R}\right]$

This means that in a dynamic calculation we have a consistency check, in that we can calculate the time constant $\alpha$ two different ways. TART always includes this consistency check in its source and criticality calculations.

\section{Analog and Expected Results}

I (DEC) have recently been surprised to find out how many people have no idea what the difference is between an analog and an expected Monte Carlo calculation. Let me give a simple example to try and explain the difference. Consider planar geometry where we have neutrons incident normally onto a slab of material 1 mean free path thick, so that in passing through the slab on average a neutron will only have one collision. We will divide the slab into 100 thin layers of material and we want to calculate results versus depth into the slab. In an analog calculation visualize a neutron incident on the slab, it has a collision in the 57-th of the 100 layers, scatters, and then emerges from the far side of the slab. From this neutron the contribution to the analog solution is a reaction, and energy deposit ONLY in the 57-th layer. But in transiting the 100 layers the neutron produced a track length, or flux, through each of the 100 layers. Based on this flux we can estimate what we expect to occur in each of the 100 layers, for anything that is a linear function of the flux, e.g., reactions, energy deposition, dose, etc. Let's compare what we learned from this one neutron: 1) for the analog result we only produced one result in the 57-th layer, 2) for the expected result we produce results in each of the 100 
layers. Furthermore the one analog result will be a sample from a distribution, e.g., the energy deposition due to scattering through 37 degrees, which will have a large statistical uncertainty. In comparison the expected results are all based on the correct average value of the distribution, e.g., the average energy deposition. Needless to say generally the expected results converge much faster than the analog results.

In a Monte Carlo calculation expected results can be obtained using the same method that deterministic codes use, which is to first determine the flux, and then use this to define reaction rates, energy deposition, etc. That is exactly what TART [1] does on the fly: for each analog event as a neutron moves from point $A$ to point $B$ in a system, it records the analog results at point $\mathrm{B}$, but it also records expected results along the entire track from point A to point B. The use of expected results is one reason why TART generally converges so quickly. It is worth noting that as geometry becomes more detailed and complicated, the probability of a neutron actually having an analog collision in a given zone decreases, making it more difficult to obtain converged analog results. In contrast this does not affect expected results nearly as much, since the neutrons still have a good probability of at least passing through each zone (needed for expected results) even if they do not have a collision in the zone (needed for analog results).

In all types of calculations, source or criticality, TART output includes both analog and expected results for rates, average times, and numbers of events. Agreement between the analog and expected results is an excellent indicator of convergence. For all of the above examples, calculations were run sufficiently long to achieve agreement between analog and expected results. 
The below table illustrates this output for a TART calculation. Notice that everything is normalized per neutron removed. With this normalization from this table we can read important quantities such as, removal time 4.56113E-03 usec, K-eff which is the production per neutron removed $1.660008 \mathrm{E}+00$, or alpha which is the net total rate $1.44718 \mathrm{E}+02 / \mathrm{usec}$, to give but a few examples.

\begin{tabular}{|c|c|c|c|}
\hline & $\begin{array}{l}\text { Rates } \\
\text { ( } 1 \text { /usec) }\end{array}$ & $\begin{array}{l}\text { Times } \\
\text { (usec) }\end{array}$ & Counts \\
\hline Removal & $2.19244 \mathrm{E}+02$ & 4.56113E-03 & $1.00000 \mathrm{E}+00$ \\
\hline Absorption (abs) & $1.54659 \mathrm{E}+02$ & $6.46583 \mathrm{E}-03$ & $7.05420 \mathrm{E}-01$ \\
\hline Leakage & $6.45849 \mathrm{E}+01$ & $1.54835 \mathrm{E}-02$ & $2.94580 \mathrm{E}-01$ \\
\hline Production (prod) & $3.63962 \mathrm{E}+02$ & $2.74754 \mathrm{E}-03$ & $1.66008 \mathrm{E}+00$ \\
\hline Net Collision (prod-abs) & $2.09303 \mathrm{E}+02$ & $4.77776 \mathrm{E}-03$ & $9.54658 \mathrm{E}-01$ \\
\hline Net Total (prod-removal) & $1.44718 \mathrm{E}+02$ & $6.90998 \mathrm{E}-03$ & $6.60078 \mathrm{E}-01$ \\
\hline \multicolumn{4}{|c|}{ Expected Rates, Times, and Counts per Removed Neutron } \\
\hline & $\begin{array}{l}\text { Rates } \\
\text { (1/usec) }\end{array}$ & $\begin{array}{l}\text { Times } \\
\text { (usec) }\end{array}$ & Counts \\
\hline Removal & $2.19244 \mathrm{E}+02$ & $4.56113 \mathrm{E}-03$ & $1.00000 \mathrm{E}+00$ \\
\hline Absorption (abs) & $1.54659 \mathrm{E}+02$ & $6.46583 E-03$ & $7.05420 \mathrm{E}-01$ \\
\hline Leakage & $6.45849 \mathrm{E}+01$ & $1.54835 \mathrm{E}-02$ & $2.94580 \mathrm{E}-01$ \\
\hline Production (prod) & $3.63962 \mathrm{E}+02$ & $2.74754 \mathrm{E}-03$ & $1.66008 \mathrm{E}+00$ \\
\hline Net Collision (pro & $2.09303 \mathrm{E}+02$ & $4.77776 \mathrm{E}-03$ & $9.54658 \mathrm{E}-01$ \\
\hline Net Total (prod-removal) & $1.44718 \mathrm{E}+02$ & $6.90998 \mathrm{E}-03$ & $6.60078 \mathrm{E}-01$ \\
\hline
\end{tabular}


University of California

Lawrence Livermore National Laboratory

Technical Information Department

Livermore, CA 94551

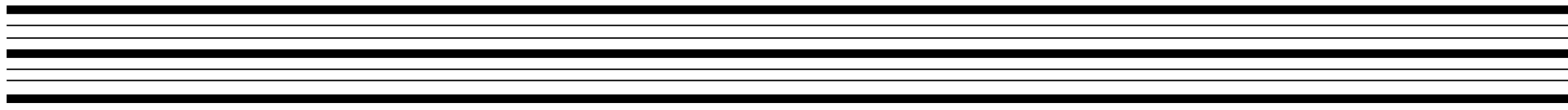

